\title{
Exactly solvable Madelung fluid and complex Burgers equations: a quantum Sturm-Liouville connection
}

\author{
Şirin A. Büyükaşık • Oktay K. Pashaev
}

Received: 23 April 2012 / Accepted: 15 July 2012 / Published online: 28 July 2012

(C) Springer Science+Business Media, LLC 2012

\begin{abstract}
Quantum Sturm-Liouville problems introduced in our paper (Büyükaşık et al. in J Math Phys 50:072102, 2009) provide a reach set of exactly solvable quantum damped parametric oscillator models. Based on these results, in the present paper we study a set of variable parametric nonlinear Madelung fluid models and corresponding complex Burgers equations, related to the classical orthogonal polynomials of Hermite, Laguerre and Jacobi types. We show that the nonlinear systems admit direct linearazation in the form of Schrödinger equation for a parametric harmonic oscillator, allowing us to solve exactly the initial value problems for these equations by the linear quantum Sturm-Liouville problem. For each type of equations, dynamics of the probability density and corresponding zeros, as well as the complex velocity field and related pole singularities are studied in details.
\end{abstract}

Keywords Schroedinger equation - Damped parametric harmonic oscillator . Sturm-Liouville problems · Quantum hydrodynamics · Madelung fluid ·

Burgers equation · Pole dynamics · Time variable parameters · Exact solvability

\section{Introduction}

Very soon after E. Schrödinger derived the fundamental equation of quantum mechanics, E. Madelung proposed a representation of the complex wave function in terms of modulus and phase $\Psi=R \exp (i S / \hbar)$, transforming the linear Schrödinger

\footnotetext{
Ş. A. Büyükaşık (凶) · O. K. Pashaev

Department of Mathematics, Izmir Institute of Technology,

35430 Urla, Izmir, Turkey

e-mail: sirinatilgan@iyte.edu.tr

O. K. Pashaev

e-mail: oktaypashaev@iyte.edu.tr
} 
equation into a system of nonlinear hydrodynamic-like equations, known as the Madelung fluid equations [1]. Madelung introduced his equations as an alternative formulation of the Schrödinger equation in order to clarify the meaning of electron and it turns out that a long time Madelung representation has provided a basis for numerous classical interpretations of quantum mechanics [2]. However, besides interpretation of quantum theory, the Madelung fluid representation becomes fundamental tool in description of quantum fluids and nonlinear evolution equations.

As a hydrodynamical formulation of quantum dynamics, it has attracted remarkable attention in the development and application of quantum trajectories as a computational tool for solving non-stationary quantum problems [4]. In this approach instead of following individual particles quantum trajectory with velocities generated by the wave function, as a solution of time-dependent Schrödinger equation, both trajectories and hydrodynamic fields are computed by fluid dynamics computational techniques applied to quantum fluid. This method has been applied to problems of phase space dynamics for open systems, mixed quantum-classical dynamics and electronic nonadiabatic energy transfer $[3,4]$. In addition to application of both the Eulerian and the Lagrangian pictures and utilization the descriptive terminology of fluid dynamics, it provides nontraditional computational techniques for solving quantum dynamics. The predictive power of this method is equivalent to traditional quantum mechanics, but may give a very economical way of solving time-dependent Schrödinger equation. Moreover, it could also stimulate a new intuitive inside to quantum dynamics and improve numerical algorithms for treating quantum systems.

Another important field, where the Madelung fluid representation becomes fundamental is the superconductivity theory [5]. In description of quantum fluids like superfluid He [6], the Madelung hydrodynamic variables have direct physical meaning, such that $\rho=|\Psi|^{2}$ plays the role of the superfluid density, and $v=\operatorname{Im}(\nabla \Psi / \Psi)$ is the superfluid velocity. Recently, the Madelung fluid representation of quantum systems becomes also an important tool for studying the dispersionless limit of nonlinear partial differential equations with envelope solitons, [7,8]. In fact it is the only way to rigorous formulation of the semiclassical limit of the one-dimensional Schrödinger equation with cubic and derivative nonlinearities before shock appearance $[8,9]$.

The quantum hydrodynamics description is an important tool also in quantum chemistry as a solution to chemical kinetic systems derived from the Schrödinger equation by the Madelung representation. This representation allows one to extend the Schrödinger equation to the rotational motions. It reduces to the Schrödinger equation for the fluid with only irrotational flow, and allows a class of wave functions whose quantum phase possesses singularity. Due to high level of complexity of chemical problems, this type of generalizations could be useful in modeling molecular behavior and chemical reactions. Quantum hydrodynamics becomes essential field of activity in theoretical chemical dynamics [4]. The idea is to employ the time-dependent Schrödinger equation to solve chemical systems for the hydrodynamic properties. In context of quantum hydrodynamics formulation it is possible to solve the chemical dynamics of several reaction mechanisms known to have pathways dominated by quantum tunneling regimes. These systems include proton transfer reactions, conformational inversions and proton-coupled electron transfer reactions [10]. The wave model approach to time dependent problems of quantum chemistry describes electrons 
as "clouds" moving in orbitals, and representing their positions by probability distribution. By this assumption of particles as "quantum fluid" it is easy to calculate the physical parameters of the system containing large number of particles in a very good agreement with experimental observations. Applicability of hydrodynamic viewpoint of quantum mechanics proved to be valid for molecular structures and chemical bonds. Individual chemical bonds were modeled by using electron flux as the "electron flow" around sodium and chloriue nuclei in [11], and used $\mathrm{H}_{2}^{+}$as a model system in [12]. In [4] the quantum trajectory method was used to model exothermic chemical reactions and some photodissociation processes.

In the last decades we observe an increasing interest in the quantum mechanical formulation of dissipative systems. One of the direct approaches to this is studied via dissipative Madelung hydrodynamics by adding a friction force, influencing the quantum particle moving in a dissipative environment [13]. This leads to quantum diffusion, describing a wave packet spreading in a dissipative environment at zero temperature. Another approach is related with self-adjoint quantization of damped oscillator, leading to the Schrödinger equation with time-dependent Hamiltonian. The most popular here is the damped quantum oscillator with constant friction, imitated by exponentially growing mass term in the Schrödinger equation and known as the Caldirola-Kanai oscillator $[14,15]$. It admits exact solutions and was extensively used to study dissipation in quantum mechanics; for review one can see [16]. After this, several methods have been developed for quantizing the damped oscillator [17-21]. At the same time quantum description of dissipation has been applied in many different areas as: molecular physics, quantum chemistry, quantum optics and plasma physics where quantum-mechanical effects are treated by means of time-dependent oscillator, $[19,22]$. It is remarkable that variable parametric Schrödinger equation for harmonic oscillator was proposed long time ago by Sakharov as descriptive of quantum cosmological models at early stage of the Universe, [23]. In all these approaches, the emergency of Madelung representation of dissipative systems arises naturally. It is related with the fact that the real and imaginary parts of the complex wave function $\Psi$ don't have direct physical meaning, but only the probability density $\rho=|\Psi|^{2}$ can be directly interpreted.

The Madelung fluid representation appearing as a dual description of the same realistic quantum system in terms of quantum hydrodynamic variables, provides an interesting tool to study the nonlinear dynamics of quantum fluids with dissipation. From mathematical point of view, we emphasize a remarkable property concerning integrability of the Madelung models. Since Madelung transform of the linear Schrödinger equation leads to nonlinear Madelung systems, using an inverse Madelung transform, clearly the nonlinear Madelung models are linearizable in the form of Schrödinger equation. Then, the inverse Madelung transform is a complex linearization transform similar to the Cole-Hopf transformation [24,25], and nonlinear models admitting such type of direct linearization are called C-integrable, [26]. It follows that, Madelung fluid equations and the complex Burgers equation are nonlinear partial differential equations belonging to the class of $\mathrm{C}$-integrable models. To extend the class of exactly solvable quantum dissipative systems, in paper [27] we have introduced the so called Quantum Sturm-Liouville problem, with wide class of quantum parametric Schrödinger equations and their exact solutions in terms of classical orthogonal 
polynomials. Then, construction of the nonlinear Madelung quantum dissipative fluid representation for these equations and corresponding exact solutions may provide an interesting results on structure of nonlinear dynamics and used in development of computational methods as quantum trajectories.

Motivated by these ideas, in [28], we have constructed a Madelung fluid model and its exact solutions for the Caldirola-Kanai quantum damped harmonic oscillator, $[14,15]$. It was shown that, probability density functions of the model behave like delta-convergent sequences at time infinity. In fact, since a solution $\Psi(q, t)$ of this model has merging zeros and describes collapse of the wave function at time infinity, then a solution $\Psi^{*}(q,-t)$ of the amplified dual system will be an expanding wave with creation of zeros as point particles from initial singularity. This evolution simulates quantum mechanism similar to creation of expanding Universe from initial singularity in Big-Bang cosmology.

In the present work, based on $[27,28]$, we introduce quantum parametric HamiltonJacobi system, parametric Madelung fluid and complex Burgers equations, linearizable in terms of Schrödinger equations for harmonic oscillator with variable parameters and related with the classical orthogonal polynomials. Quantization of the singular Sturm-Liouville problems studied in [27] provides a reach set of exactly solvable harmonic oscillator models with time dependent parameters, and allows us to obtain exact solutions of the corresponding Madelung models with variable parameters. Here, we study Madelung fluid and complex Burgers equations of Hermite, Laguerre and Jacobi type associated with the corresponding orthogonal polynomials. For each type of these equations with specific time dependent damping, exact solutions and dynamics of the zeros of the probability density, as well as dynamics of the pole singularities of the complex velocity are studied in details.

\section{Madelung fluid: a quantum Sturm-Liouville connection}

Quantum Sturm-Liouville problems were introduced in [27] and Madelung representation of Schrödinger equation for harmonic oscillator with variable parameters was discussed in [28]. In this preliminary section, we establish their connection and provide the basic equations. Consider the second order differential equation

$$
\frac{d}{d t}\left[\mu(t) \frac{d}{d t}\right] x(t)+[\lambda r(t)] x(t)=0, \quad a<t<b,
$$

where $\lambda$ is a spectral parameter, $\mu(t)>0$ on $(a, b), \mu(t) \in C^{1}(a, b)$, and $\lambda r(t)>0$ on $(a, b), r(t) \in C(a, b)$. Let singularities be allowed at the end points of the fundamental domain $(a, b)$, such as (i) $(a, b)$ is an infinite domain, (ii) $\mu(a)=0$ or/and $\mu(b)=0$, (iii) mixture of the first two cases. Then, Eq. (1) together with boundary conditions like $x(t)$ must be continuous or bounded or become infinite of an order less than the prescribed, defines a singular Sturm-Liouville problem, [29]. On the other 
hand, Eq. (1) can be written in the form of damped parametric oscillator

$$
\ddot{x}+\frac{\dot{\mu}(t)}{\mu(t)} \dot{x}+\frac{\lambda r(t)}{\mu(t)} x=0, \quad t \in(a, b),
$$

where $\Gamma(t)=\dot{\mu}(t) / \mu(t)$ is the damping coefficient and $\lambda r(t) / \mu(t)$ is the frequency. Then, for the formal self-adjoint operator $T$, one can find the action functional in the form

$S=\frac{1}{2}\langle x(t)|T| x(t)\rangle=\frac{1}{2} \int_{a}^{b} x(t) T x(t) d t=-\frac{1}{2} \int_{a}^{b}\left[\mu(t) \dot{x}^{2}(t)-\mu(t) \omega^{2}(t) x^{2}(t)\right] d t$,

assuming $\left.\mu(t) x(t) \dot{x}(t)\right|_{a} ^{b}=0$. This leads to Lagrangian $L=\mu(t)\left[\dot{x}^{2}(t)-\right.$ $\left.\omega^{2}(t) x^{2}(t)\right] / 2$, and classical Hamiltonian

$$
H(x, p)=\frac{p^{2}}{2 \mu(t)}+\frac{\mu(t) \omega^{2}(t)}{2} x^{2},
$$

where $p=\partial L / \partial \dot{x}=\mu(t) \dot{x}(t)$. Then, as a result of self-adjoint quantization one obtains an explicitly time-dependent quantum Hamiltonian

$$
\hat{H}(t)=-\frac{\hbar^{2}}{2 \mu(t)} \frac{\partial^{2}}{\partial q^{2}}+\frac{\lambda r(t)}{2} \hat{q}^{2} .
$$

This procedure allows us to study Schrödinger equations for harmonic oscillator related with the singular Sturm-Liouville problems and the classical orthogonal polynomials, known as quantum Sturm-Liouville problems, [27]. For generality, we will use notation $\lambda r(t) \equiv \mu(t) \omega^{2}(t)$, and consider the IVP

$$
\begin{aligned}
i \hbar \frac{\partial \Psi}{\partial t} & =-\frac{\hbar^{2}}{2 \mu(t)} \frac{\partial^{2} \Psi}{\partial q^{2}}+\frac{\mu(t) \omega^{2}(t)}{2} q^{2} \Psi, \\
\Psi\left(q, t_{0}\right) & =\psi(q), \quad-\infty<q<\infty,
\end{aligned}
$$

see [27] and references there in. It is known that, if $x(t)$ is solution of the IVP for the classical parametric oscillator

$$
\ddot{x}+\frac{\dot{\mu}(t)}{\mu(t)} \dot{x}+\omega^{2}(t) x=0, \quad x\left(t_{0}\right)=x_{0} \neq 0, \quad \dot{x}\left(t_{0}\right)=0,
$$

with $\mu(t)>0, \mu(t) \in C^{\prime}(a, b), \omega^{2}(t)>0, \omega(t) \in C(a, b), t_{0} \in(a, b)$, then the solution of the quantum evolution problem (2-3) is formally given by $\Psi(q, t)=$ $\widehat{U}\left(t, t_{0}\right) \psi(q)$, where the evolution operator is

$$
\widehat{U}\left(t, t_{0}\right)=\exp \left(\frac{i}{2} f(t) q^{2}\right) \exp \left(h(t)\left(q \frac{\partial}{\partial q}+\frac{1}{2}\right)\right) \exp \left(-\frac{i}{2} g(t) \frac{\partial^{2}}{\partial q^{2}}\right)
$$


and the auxiliary functions are

$$
\begin{aligned}
& f(t)=\frac{\mu(t)}{\hbar} \frac{\dot{x}(t)}{x(t)} ; \quad g(t)=-\hbar x^{2}\left(t_{0}\right) \int^{t} \frac{d \xi}{\mu(\xi) x^{2}(\xi)}, \quad g\left(t_{0}\right)=0 ; \\
& h(t)=\ln \frac{\left|x\left(t_{0}\right)\right|}{|x(t)|} .
\end{aligned}
$$

If the initial function is given as

$$
\Psi\left(q, t_{0}\right)=\varphi_{k}(q)=N_{k} e^{-\frac{\Omega_{0}}{2} q^{2}} H_{k}\left(\sqrt{\Omega_{0}} q\right), \quad k=0,1,2, \ldots,
$$

where $\varphi_{k}(q)$ are the normalized eigenfunctions of the Hamiltonian for the standard harmonic oscillator $\hat{H}_{0}=-\left(\hbar^{2} / 2\right) \partial_{q}^{2}+\left(\omega_{0}^{2} / 2\right) q^{2}, \omega_{0}=$ const, $\Omega_{0}=\omega_{0} / \hbar$, then the time-evolved state becomes

$$
\begin{aligned}
\Psi_{k}(q, t)= & N_{k} \sqrt{R(t)} \times \exp \left(i\left(k+\frac{1}{2}\right) \arctan \left(\Omega_{0} g(t)\right)\right) \\
& \times \exp \left(i\left(\frac{\mu(t) \dot{x}(t)}{2 \hbar x(t)}-\frac{\Omega_{0}^{2}}{2} g(t) R^{2}(t)\right) q^{2}\right) \times \exp \left(-\frac{\Omega_{0}}{2} R^{2}(t) q^{2}\right) \\
& \times H_{k}\left(\sqrt{\Omega_{0}} R(t) q\right),
\end{aligned}
$$

where

$$
R(t)=\left(\frac{x_{0}^{2}}{x^{2}(t)+\left(\Omega_{0} x(t) g(t)\right)^{2}}\right)^{\frac{1}{2}}
$$

It follows that, the corresponding probability density $\rho_{k}(q, t)=\left|\Psi_{k}(q, t)\right|^{2}$ is of the form

$$
\begin{aligned}
\rho_{k}(q, t) & =\frac{1}{2^{k} k ! \sqrt{\pi}} \times \sqrt{\Omega_{0}} R(t) \times \exp \left(-\left(\sqrt{\Omega_{0}} R(t) q\right)^{2}\right) \times H_{k}^{2}\left(\sqrt{\Omega_{0}} R(t) q\right), \\
k & =0,1,2, \ldots
\end{aligned}
$$

Following Madelung's idea we write the complex-valued wave function in polar form

$$
\Psi(q, t)=\sqrt{\rho(q, t)} \exp \left(\frac{i}{\hbar} S(q, t)\right)=\exp \left(\frac{1}{2} \ln \rho(q, t)+\frac{i}{\hbar} S(q, t)\right)
$$

where $\rho(q, t) \geq 0$ is the probability density and $S(q, t)$ is the action, both being realvalued functions. Introducing this representation in Schrödinger equation (2) leads to 
a system of nonlinear coupled partial differential equations,

$$
\left\{\begin{array}{l}
\frac{\partial S}{\partial t}+\frac{1}{2 \mu(t)}\left(\frac{\partial S}{\partial q}\right)^{2}+\frac{\mu(t) \omega^{2}(t)}{2} q^{2}=\frac{\hbar^{2}}{2 \mu(t)}\left[\frac{1}{\sqrt{\rho}} \frac{\partial^{2} \sqrt{\rho}}{\partial q^{2}}\right] \\
\frac{\partial \rho}{\partial t}+\frac{\partial}{\partial q}\left[\rho \frac{1}{\mu(t)} \frac{\partial S}{\partial q}\right]=0
\end{array}\right.
$$

so that the linear dynamics in complex plane is projected onto a nonlinear dynamics in real space. The first equation is a variable parametric quantum Hamilton-Jacobi equation, where the term with explicit $\hbar$ dependence is a nonstationary quantum potential

$$
Q(q, t)=-\frac{\hbar^{2}}{2 \mu(t)}\left[\frac{1}{\sqrt{\rho}} \frac{\partial^{2} \sqrt{\rho}}{\partial q^{2}}\right]
$$

encoding the quantum aspects of the theory. The second equation is a continuity equation for the probability density. Then, using (11), one finds that, system (12) with real-valued initial conditions $S\left(q, t_{0}\right)=\tilde{S}(q), \quad \rho\left(q, t_{0}\right)=\tilde{\rho}(q) \geq 0$, has formal solution

$$
S(q, t)=-i \hbar \ln \left(\frac{\Psi(q, t)}{|\Psi(q, t)|}\right), \quad \rho(q, t)=|\Psi(q, t)|^{2},
$$

where $\Psi(q, t)$ is a solution of the Schrödinger equation (2) with initial condition

$$
\Psi\left(q, t_{0}\right)=\sqrt{\tilde{\rho}(q)} \exp \left(\frac{i}{\hbar} \tilde{S}(q)\right) .
$$

We remark that, in general the action $S(q, t)=-i \hbar \ln (\Psi /|\Psi|)+2 \pi n \hbar, n=$ $0, \pm 1, \pm 2, \ldots$ is a multi-valued function, but fixing the initial condition $S\left(q, t_{0}\right)=$ $\tilde{S}(q)$ leads to a single-valued solution of the IVP. Clearly, the explicit form of the solutions $S(q, t)$ and $\rho(q, t)$ depends on the properties of the initial functions $\tilde{\rho}(q)$ and $\tilde{S}(q)$. In this work, we shall consider initial functions $\tilde{\rho}(q), \tilde{S}(q)$ so that in (14) one has $\Psi\left(q, t_{0}\right) \in L_{2}(R)$. Specifically, in the following sections, for all particular problems the initial conditions will be taken such that $\Psi\left(q, t_{0}\right)=\varphi_{k}(q) \in L_{2}(R)$, where $\varphi_{k}(q)$ is given by (7).

Next, introducing velocity field $v(q, t)=(1 / \mu(t))(\partial S / \partial q)$, system (12) transforms to a system of parametric Madelung fluid equations with dissipation

$$
\left\{\begin{array}{l}
\frac{\partial v}{\partial t}+\frac{\dot{\mu}(t)}{\mu(t)} v+v \frac{\partial v}{\partial q}=-\frac{1}{\mu(t)} \frac{\partial}{\partial q}\left[\frac{-\hbar^{2}}{2 \mu(t)}\left(\frac{1}{\sqrt{\rho}} \frac{\partial^{2} \sqrt{\rho}}{\partial q^{2}}\right)+\frac{\mu(t) \omega^{2}(t)}{2} q^{2}\right] \\
\frac{\partial \rho}{\partial t}+\frac{\partial}{\partial q}[\rho v]=0
\end{array}\right.
$$

where $\Gamma(t)=\dot{\mu}(t) / \mu(t)$ is the friction coefficient, which for nonconstant $\mu(t)$ reflects the dissipative nature of the system. As given in [28], system (15) with real-valued initial conditions $v\left(q, t_{0}\right)=\tilde{v}(q), \rho\left(q, t_{0}\right)=\tilde{\rho}(q) \geq 0$, has formal solution 


$$
v(q, t)=-\frac{i \hbar}{\mu(t)} \frac{\partial}{\partial q} \ln \left(\frac{\Psi(q, t)}{|\Psi(q, t)|}\right), \quad \rho(q, t)=|\Psi(q, t)|^{2},
$$

where $\Psi(q, t)$ is solution of the Schrödinger equation (2) with initial condition

$$
\Psi\left(q, t_{0}\right)=\sqrt{\tilde{\rho}(q)} \exp \left(\frac{i}{\hbar} \mu\left(t_{0}\right) \int^{q} \tilde{v}(\xi) d \xi\right) .
$$

Writing the wave function in the form $\Psi(q, t)=\exp \left(\frac{i}{\hbar} \mu(t) F(q, t)\right)$, where $F(q, t)$ is a complex potential, the linear Schrödinger equation (2) transforms to a parametric potential complex Burgers equation. Then, the corresponding IVP

$$
\left\{\begin{array}{l}
\frac{\partial F}{\partial t}+\frac{\dot{\mu}(t)}{\mu(t)} F+\frac{1}{2}\left(\frac{\partial F}{\partial q}\right)^{2}+\frac{\omega^{2}(t)}{2} q^{2}=\frac{i \hbar}{2 \mu(t)} \frac{\partial^{2} F}{\partial q^{2}} \\
F\left(q, t_{0}\right)=\widetilde{F}(q)
\end{array}\right.
$$

has formal solution given by $F(q, t)=-i \hbar(\ln \Psi(q, t)) / \mu(t)$, where $\Psi(q, t)$ is solution of the Schrödinger equation (2) subject to the initial condition $\Psi\left(q, t_{0}\right)=$ $\exp \left((i / \hbar) \mu\left(t_{0}\right) \widetilde{F}(q)\right)$.

Representation of the wave function in the form

$$
\Psi(q, t)=\exp \left(\frac{i}{h} \mu(t) \int^{q} V(\xi, t) d \xi\right)
$$

where $V(q, t)$ is a complex velocity, transforms Schrödinger equation (2) to a complex Burgers equation with time dependent parameters. Then, the general IVP for the complex Burgers equation

$$
\left\{\begin{array}{l}
\frac{\partial V}{\partial t}+\frac{\dot{\mu}(t)}{\mu(t)} V+V \frac{\partial V}{\partial q}+\omega^{2}(t) q=\frac{i \hbar}{2 \mu(t)} \frac{\partial^{2} V}{\partial q^{2}}, \\
V\left(q, t_{0}\right)=\widetilde{V}(q),
\end{array}\right.
$$

has formal solution given by the complex Cole-Hopf transformation

$$
V(q, t)=-\frac{i \hbar}{\mu(t)} \frac{\partial}{\partial q}(\ln \Psi(q, t))
$$

where $\Psi(q, t)$ is solution of the Schrödinger equation (2) with the initial condition

$$
\Psi\left(q, t_{0}\right)=\exp \left(\frac{i}{\hbar} \mu\left(t_{0}\right) \int^{q} \widetilde{V}(\xi) d \xi\right)
$$




\section{Hermite type Madelung fluid and complex Burgers equation}

Now, we introduce and obtain exact explicit solutions of quantum Hamilton-Jacobi system, Madelung hydrodynamic model and complex Burgers equation related with the Sturm-Liouville problem for the classical Hermite polynomials. For this, we start by choosing an exponentially decreasing mass $\mu(t)=e^{-t^{2}}$, and constant frequency $\omega^{2}(t)=2 n, t \in(-\infty, \infty)$. This leads to the following systems.

A. First, we have Hermite type quantum Hamilton-Jacobi system

$$
\left\{\begin{array}{l}
\frac{\partial S}{\partial t}+\frac{e^{t^{2}}}{2}\left(\frac{\partial S}{\partial q}\right)^{2}+n e^{-t^{2}} q^{2}=\frac{\hbar^{2} e^{t^{2}}}{2}\left[\frac{1}{\sqrt{\rho}} \frac{\partial^{2} \sqrt{\rho}}{\partial q^{2}}\right], \quad n=0,1,2, \ldots \\
\frac{\partial \rho}{\partial t}+\frac{\partial}{\partial q}\left[e^{t^{2}} \rho \frac{\partial S}{\partial q}\right]=0
\end{array}\right.
$$

Under the transformation

$$
S(q, t)=-i \hbar \ln \left(\frac{\Psi(q, t)}{|\Psi(q, t)|}\right), \quad \rho(q, t)=|\Psi(q, t)|^{2},
$$

it converts to the linear Hermite type Schrödinger equation

$$
i \hbar \frac{\partial \Psi}{\partial t}=-\hbar^{2} \frac{e^{t^{2}}}{2} \frac{\partial^{2}}{\partial q^{2}} \Psi+n e^{-t^{2}} q^{2} \Psi
$$

where $\mu(t)=e^{-t^{2}}$ and $\omega^{2}(t)=2 n, t \in(-\infty, \infty), n=0,1,2, \ldots$ Then, system (21), with real-valued initial conditions $S\left(q, t_{0}\right)=\tilde{S}(q), \quad \rho\left(q, t_{0}\right)=\tilde{\rho}(q), \tilde{\rho}(q) \geq 0$ has formal solution given by (22), where $\Psi(q, t)$ is solution of Eq. (23) with initial condition $\Psi\left(q, t_{0}\right)=\sqrt{\tilde{\rho}(q)} \exp ((i / \hbar) \tilde{S}(q))$.

In particular, when the initial state is given by (7), i.e. $\Psi\left(q, t_{0}\right)=\varphi_{k}(q)$, solution of (23), see [27], is known as,

$$
\begin{aligned}
\Psi_{n, k}(q, t)= & N_{k} \sqrt{R_{n}(t)} \times \exp \left(\frac{i}{2 \hbar}\left(e^{-t^{2}} \frac{\dot{H}_{n}(t)}{H_{n}(t)}\right) q^{2}\right) \times \exp \left(-\frac{i}{2} \Omega_{0}^{2} g_{n}(t) R_{n}^{2}(t) q^{2}\right) \\
& \times \exp \left(i\left(k+\frac{1}{2}\right) \arctan \left(\Omega_{0} g_{n}(t)\right)\right) \times \exp \left(-\frac{\Omega_{0}}{2} R_{n}^{2}(t) q^{2}\right) \\
& \times H_{k}\left(\sqrt{\Omega_{0}} R_{n}(t) q\right), \quad k=0,1,2, \ldots
\end{aligned}
$$

where $H_{n}(t), n=0,1,2, \ldots$, are the Hermite polynomials, satisfying

$$
\ddot{H}_{n}-2 t \dot{H}_{n}+2 n H_{n}=0, \quad H_{n}\left(t_{0}\right) \neq 0, \quad \dot{H}_{n}\left(t_{0}\right)=0
$$

for proper choice of $t_{0} \in(-\infty, \infty)$, (indeed, to have the even Hermite polynomials, in (25) one can take $t_{0}=0$, since the initial conditions $H_{n}(0) \neq 0$ and $\dot{H}_{n}(0)=0$ 
hold for even $n$, and to have odd Hermite polynomials, there exists $t_{0}$ such that these initial conditions are again satisfied). The other auxiliary functions in (24) are

$$
\begin{aligned}
& g_{n}(t)=-\hbar H_{n}^{2}\left(t_{0}\right) \int^{t} \frac{e^{\xi^{2}} d \xi}{H_{n}^{2}(\xi)}, \quad g\left(t_{0}\right)=0 ; \\
& R_{n}(t)=\left(\frac{H_{n}^{2}\left(t_{0}\right)}{H_{n}{ }^{2}(t)+\left[\Omega_{0} g_{n}(t) H_{n}(t)\right]^{2}}\right)^{\frac{1}{2}} .
\end{aligned}
$$

Therefore, system (21) with specific initial conditions

$S_{k}\left(q, t_{0}\right)=0, \quad \rho_{k}\left(q, t_{0}\right)=N_{k}^{2} \exp \left(-\left(\sqrt{\Omega_{0}} q\right)^{2}\right) H_{k}^{2}\left(\sqrt{\Omega_{0}} q\right), \quad k=0,1,2, \ldots$

has exact solutions

$S_{n, k}(q, t)=\frac{1}{2}\left(e^{-t^{2}} \frac{\dot{H}_{n}(t)}{H_{n}(t)}-\hbar \Omega_{0}^{2} g_{n}(t) R_{n}^{2}(t)\right) q^{2}+\hbar\left(k+\frac{1}{2}\right) \arctan \left(\Omega_{0} g_{n}(t)\right)$,

and

$$
\rho_{n, k}(q, t)=N_{k}^{2} \times R_{n}(t) \times \exp \left(-\left(\sqrt{\Omega_{0}} R_{n}(t) q\right)^{2}\right) \times H_{k}^{2}\left(\sqrt{\Omega_{0}} R_{n}(t) q\right),
$$

where $g_{n}(t)$ and $R_{n}(t)$ are defined by (26).

B. Introducing the velocity field $v(q, t)=e^{t^{2}} \partial S / \partial q, t \in(-\infty, \infty)$ in system (21), or setting the specific parameters $\mu(t)=e^{-t^{2}}$ and $\omega^{2}(t)=2 n$ in (15), we obtain Hermite type Madelung hydrodynamic equations

$$
\left\{\begin{array}{l}
\frac{\partial v}{\partial t}-2 t v+v \frac{\partial v}{\partial q}=-e^{t^{2}} \frac{\partial}{\partial q}\left[\frac{-\hbar^{2}}{2} e^{t^{2}}\left(\frac{1}{\sqrt{\rho}} \frac{\partial^{2} \sqrt{\rho}}{\partial q^{2}}\right)+n e^{-t^{2}} q^{2}\right], \\
\frac{\partial \rho}{\partial t}+\frac{\partial}{\partial q}[\rho v]=0,
\end{array}\right.
$$

with friction coefficient $\Gamma(t)=-2 t$. Using transformation

$$
v(q, t)=-i \hbar e^{t^{2}} \frac{\partial}{\partial q} \ln \left(\frac{\Psi(q, t)}{|\Psi(q, t)|}\right), \quad \rho(q, t)=|\Psi(q, t)|^{2},
$$

system (28) is linearizable in the form of Hermite type Schrödinger equation (23). Therefore, the system of Madelung fluid Eq. (28) with general initial conditions $v\left(q, t_{0}\right)=\tilde{v}(q), \rho\left(q, t_{0}\right)=\tilde{\rho}(q)$, has formal solution given by (29), where $\Psi(q, t)$ 


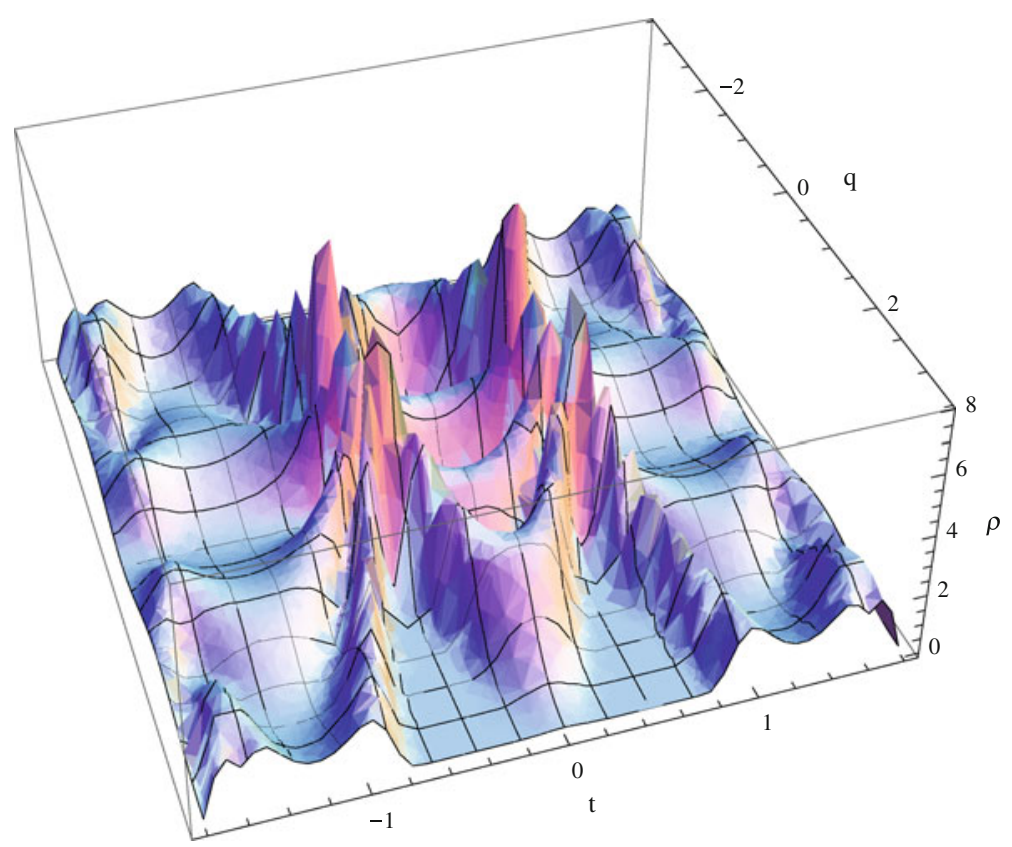

Fig. 1 Hermite type model. Probability density $\rho_{4,3}(q, t)$. for $n=4, k=3$

is solution of the Schrödinger equation (23) with initial condition

$$
\Psi\left(q, t_{0}\right)=\sqrt{\tilde{\rho}(q)} \exp \left(\frac{i}{\hbar} e^{-t_{0}^{2}} \int^{q} \tilde{v}(\xi) d \xi\right)
$$

It follows that, the IVP for system (28) with specific initial conditions

$v_{k}\left(q, t_{0}\right)=0, \quad \rho_{k}\left(q, t_{0}\right)=N_{k}^{2} \exp \left(-\left(\sqrt{\Omega_{0}} q\right)^{2}\right) H_{k}^{2}\left(\sqrt{\Omega_{0}} q\right), \quad k=0,1,2, \ldots$

has exact solutions

$$
v_{n}(q, t)=\left(\frac{\dot{H}_{n}(t)}{H_{n}(t)}-\hbar \Omega_{0}^{2} e^{t^{2}} g_{n}(t) R_{n}^{2}(t)\right) q, \quad \forall k=0,1,2, \ldots
$$

and $\rho_{n, k}(q, t)$ found in (27). As an illustrative example, in Fig. 1 we construct the plot of the probability density $\rho_{n, k}(q, t)$ for the case $n=4, k=3,\left(\hbar=\omega_{0}^{2}=x_{0}=1\right.$ and $\left.t_{0}=0\right)$.

C. The potential complex Burgers equation of Hermite type is of the form

$$
\frac{\partial F}{\partial t}-2 t F+\frac{1}{2}\left(\frac{\partial F}{\partial q}\right)^{2}+n q^{2}=\frac{i \hbar}{2} e^{t^{2}} \frac{\partial^{2} F}{\partial q^{2}}, \quad n=0,1,2, \ldots
$$


and using the transformation

$$
F(q, t)=-i \hbar e^{t^{2}}(\ln \Psi(q, t)),
$$

it converts to Hermite type Schrödinger equation (23). Then, the IVP for the potential complex Burgers equation (31) subject to the general initial condition $F\left(q, t_{0}\right)=\widetilde{F}(q)$ has formal solution given by (32), where $\Psi(q, t)$ is a solution of (23) with initial condition $\Psi\left(q, t_{0}\right)=\exp \left((i / \hbar) e^{-t_{0}^{2}} \widetilde{F}(q)\right)$. The IVP with specific initial conditions,

$$
\left\{\begin{array}{l}
\frac{\partial F}{\partial t}-2 t F+\frac{1}{2}\left(\frac{\partial F}{\partial q}\right)^{2}+n q^{2}=\frac{i \hbar}{2} e^{t^{2}} \frac{\partial^{2} F}{\partial q^{2}}, \quad n=0,1,2, \ldots \\
F_{k}\left(q, t_{0}\right)=i \hbar e^{t_{0}^{2}}\left(\frac{\Omega_{0}}{2} q^{2}-\ln \left(N_{k} H_{k}\left(\sqrt{\Omega_{0}} q\right)\right), \quad k=0,1,2, \ldots\right.
\end{array}\right.
$$

has exact solutions of the form

$$
\begin{aligned}
F_{n, k}(q, t)= & {\left[\frac{1}{2}\left(\frac{\dot{H}_{n}(t)}{H_{n}(t)}-\hbar \Omega_{0}^{2} g_{n}(t) e^{t^{2}} R_{n}^{2}(t)\right) q^{2}+\hbar e^{t^{2}}\left(k+\frac{1}{2}\right) \arctan \left(\Omega_{0} g_{n}(t)\right)\right] } \\
& +i \hbar e^{t^{2}}\left[\frac{\Omega_{0}}{2} R_{n}^{2}(t) q^{2}-\ln \left(N_{k} \sqrt{R_{n}(t)} H_{k}\left(\sqrt{\Omega_{0}} R_{n}(t) q\right)\right)\right] .
\end{aligned}
$$

Writing $F(q, t)=\mathrm{F}_{1}(q, t)+i \mathrm{~F}_{2}(q, t)$, where $\mathrm{F}_{1}$ is the velocity potential, and $\mathrm{F}_{2}$ is the stream function, both being real-valued, the potential complex Burgers equation splits into a system of coupled equations

$$
\left\{\begin{array}{l}
\frac{\partial \mathrm{F}_{1}}{\partial t}-2 t \mathrm{~F}_{1}+\frac{1}{2}\left(\left(\frac{\partial \mathrm{F}_{1}}{\partial q}\right)^{2}-\left(\frac{\partial \mathrm{F}_{2}}{\partial q}\right)^{2}\right)+n q^{2}=-\frac{\hbar}{2} e^{t^{2}} \frac{\partial^{2} \mathrm{~F}_{2}}{\partial q^{2}} \\
\frac{\partial \mathrm{F}_{2}}{\partial t}-2 t \mathrm{~F}_{2}+\frac{\partial \mathrm{F}_{1}}{\partial q} \frac{\partial \mathrm{F}_{2}}{\partial q}=\frac{\hbar}{2} e^{t^{2}} \frac{\partial^{2} \mathrm{~F}_{1}}{\partial q^{2}}
\end{array}\right.
$$

which with specific initial conditions

$$
\mathrm{F}_{1, k}\left(q, t_{0}\right)=0, \quad \mathrm{~F}_{2, k}\left(q, t_{0}\right)=\hbar e^{t_{0}^{2}}\left(\frac{\Omega_{0}}{2} q^{2}-\ln \left(N_{k} H_{k}\left(\sqrt{\Omega_{0}} q\right)\right), \quad k=0,1,2, \ldots\right.
$$

has solutions, respectively the real and imaginary parts of (34).

D. Now, we introduce the Hermite type complex Burgers equation

$$
\frac{\partial V}{\partial t}-2 t V+V \frac{\partial V}{\partial q}+2 n q=\frac{i \hbar}{2} e^{t^{2}} \frac{\partial^{2} V}{\partial q^{2}}, \quad n=0,1,2, \ldots
$$

By the generalized complex Cole-Hopf transformation

$$
V(q, t)=-i \hbar e^{t^{2}} \frac{\partial}{\partial q}(\ln \Psi(q, t))
$$




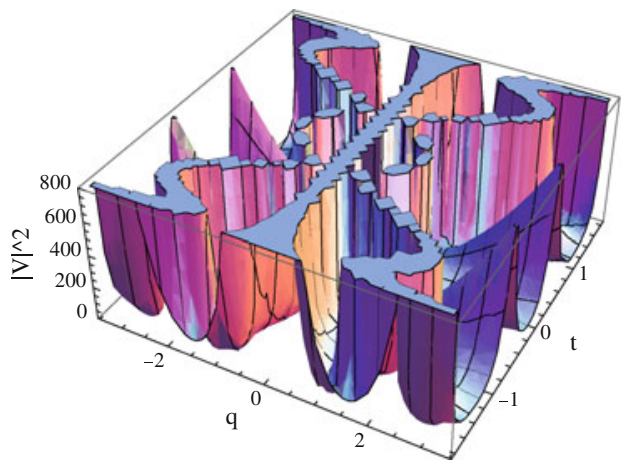

(a)

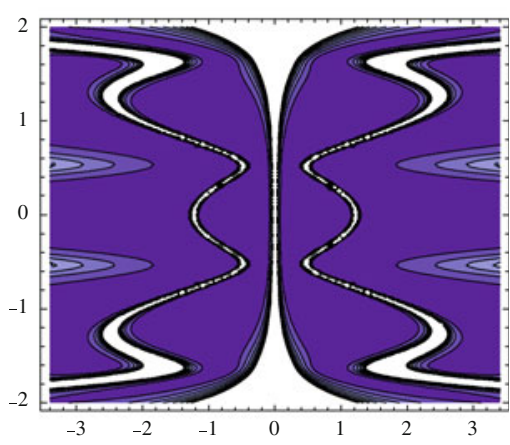

(b)

Fig. 2 Hermite type model. a Plot of $\left|V_{4,3}(q, t)\right|^{2}$. b Contour plot of $\left|V_{4,3}(q, t)\right|^{2}$

it is linearizable in the form of Schrödinger equation (23). Then, the general IVP for (36) with initial condition $V\left(q, t_{0}\right)=\widetilde{V}(q)$ has formal solution given by (37), where $\Psi(q, t)$ is solution of the IVP for the Schrödinger equation (23) with initial condition

$$
\Psi\left(q, t_{0}\right)=\exp \left(\frac{i}{\hbar} e^{-t_{0}^{2}} \int^{q} \widetilde{V}(\xi) d \xi\right) .
$$

As an example, we give the IVP with specific initial condition

$$
\begin{cases}\frac{\partial V}{\partial t}-2 t V+V \frac{\partial V}{\partial q}+2 n q=\frac{i \hbar}{2} e^{t^{2}} \frac{\partial^{2} V}{\partial q^{2}}, \quad n=0,1,2, \ldots, \\ V_{k}\left(q, t_{0}\right)=i \hbar e^{t_{0}^{2}}\left[\Omega_{0} q-\frac{\partial_{q} H_{k}\left(\sqrt{\Omega_{0}} q\right)}{H_{k}\left(\sqrt{\Omega_{0}} q\right)}\right], \quad k=0,1,2, \ldots,\end{cases}
$$

which has explicit solution

$$
\begin{aligned}
V_{n, k}(q, t)= & {\left[\frac{\dot{H}_{n}(t)}{H_{n}(t)}-\hbar \Omega_{0}^{2} e^{t^{2}} g_{n}(t) R_{n}^{2}(t)\right] q } \\
& +i \hbar e^{t^{2}}\left[\Omega_{0} R_{n}^{2}(t) q-\frac{\partial_{q} H_{k}\left(\sqrt{\Omega_{0}} R_{n}(t) q\right)}{H_{k}\left(\sqrt{\Omega_{0}} R_{n}(t) q\right)}\right] .
\end{aligned}
$$

The quantity $\left|V_{n, k}(q, t)\right|^{2}$ can be seen as an analog of kinetic energy, or envelope of the modulated wave. Note that, the complex velocity $V_{n, k}(q, t)$ has finite time singularities at zeros of $H_{n}(t)$, and it has moving singularities where $H_{k}\left(\sqrt{\Omega_{0}} R_{n}(t) q\right)=0$. In Fig. 2, we give the plot of $\left|V_{n, k}(q, t)\right|^{2}$ for $n=4, k=3,\left(\hbar=\omega_{0}^{2}=x_{0}=1, t_{0}=0\right)$ and the corresponding contour plot from which one can observe also the motion of the three pole singularities. 
Writing the complex velocity as $V(q, t)=v(q, t)+i u(q, t)$, where $v$ and $u$ are real-valued functions, the complex Burgers equation splits into real and imaginary parts

$$
\left\{\begin{array}{l}
\frac{\partial v}{\partial t}+v \frac{\partial v}{\partial q}-2 t v-u \frac{\partial u}{\partial q}+2 n q=-\frac{\hbar}{2} e^{t^{2}} \frac{\partial^{2} u}{\partial q^{2}} \\
\frac{\partial u}{\partial t}-2 t u+u \frac{\partial v}{\partial q}+v \frac{\partial u}{\partial q}=\frac{\hbar}{2} e^{t^{2}} \frac{\partial^{2} v}{\partial q^{2}}
\end{array}\right.
$$

Then, system (40) with specific initial conditions

$$
v_{k}\left(q, t_{0}\right)=0, \quad u_{k}\left(q, t_{0}\right)=\hbar e^{t_{0}^{2}}\left[\Omega_{0} q-\frac{\partial_{q} H_{k}\left(\sqrt{\Omega_{0}} q\right)}{H_{k}\left(\sqrt{\Omega_{0}} q\right)}\right], \quad k=0,1,2, \ldots
$$

has exact solutions $v_{n}(q, t)$ and $u_{n, k}(q, t)$, which are clearly the real and imaginary parts of (39), respectively. Using (11) and (20) we note the relation

$$
V(q, t)=v(q, t)+i u(q, t)=e^{t^{2}} \frac{\partial S}{\partial q}-\frac{i \hbar}{2} e^{t^{2}} \frac{\partial}{\partial q}(\ln \rho) .
$$

E. Motion of the zeros and poles. The zeros of the wave function $\Psi(q, t)$ (and $\rho(q, t))$ for the Schrödinger equation, due to the complex Cole-Hopf transformation (37), become pole singularities of the solution $V(q, t)$ (and $|V(q, t)|^{2}$ ) for the nonlinear complex Burgers equation. From (24), one can see that solution $\Psi_{n, k}(q, t)$ of the Schrödinger equation has zeros at points where $H_{k}\left(\sqrt{\Omega_{0}} R_{n}(t) q\right)=0$, and these zeros are pole singularities of the complex Burgers solution $V_{n, k}(q, t)$ given by (39). If $\tau_{k}^{(l)}, l=1,2, \ldots, k$, denote the zeros of the Hermite polynomial $H_{k}(\xi)$, then the motion of the zeros of $\Psi_{n, k}(q, t)$ and poles of $V_{n, k}(q, t)$ for fixed $n=0,1, \ldots$ and $k=1,2, \ldots$ is described by

$$
q_{k}^{(l)}(t)=\frac{\tau_{k}^{(l)}}{\sqrt{\Omega_{0}} R_{n}(t)}=\frac{\tau_{k}^{(l)}}{\sqrt{\Omega_{0}}}\left(\frac{H_{n}^{2}(t)+\left[\Omega_{0} g_{n}(t) H_{n}(t)\right]^{2}}{H_{n}^{2}\left(t_{0}\right)}\right)^{\frac{1}{2}}, \quad l=1,2, \ldots, k
$$

Clearly, $k$ is the number of the moving zeros/poles and $n$ is the number of their oscillations. At times close to $t=0$, and $n>1$, the singularities show oscillatory motion. Then, when $t \rightarrow \pm \infty$, one has $R_{n}(t) \rightarrow 0$ and $\left|q_{k}^{(l)}(t)\right| \rightarrow \infty$, showing that the zeros/poles go away from the origin $q=0$. Moreover, the distance between different zeros/poles also increases with time, that is for fixed $k$ and $i \neq j$, $\left|q_{k}^{(i)}(t)-q_{k}^{(j)}(t)\right| \rightarrow \infty$ as $t \rightarrow \pm \infty$, as an example see Fig. 2.

Finally we remark that, solutions of the equation $d q / d t=v(q, t)$ are known as quantum trajectories. As stated in [4], the patterns developed by these quantum trajectories $q(t)$ as they emanate from an ensemble of "launch points" exactly define the history of the system as it evolves from the initial to the final state. The probability 
amplitude $\sqrt{\rho}$ and phase $S$ are transported along these trajectories, and observables may be computed directly in terms of this information. Such computations are not subject of this work, but it could be interesting to see the picture of the quantum trajectories for our specific models. Thus, if $v_{n}(q, t)$ is given by (30), solving the equation $d q_{n} / d t=v_{n}(q, t)$ gives

$$
q_{n}(t)=c / R_{n}(t)=c\left(\frac{H_{n}^{2}(t)+\left[\Omega_{0} g_{n}(t) H_{n}(t)\right]^{2}}{H_{n}^{2}\left(t_{0}\right)}\right)^{\frac{1}{2}},
$$

where $R_{n}(t)$ is defined in (26), and constant $c$ is fixed by the initial position $q_{n}\left(t_{0}\right)$. If we fix $n$ and choose initial positions in terms of the zeros of the Hermite polynomial $H_{k}(\xi)$, that is $q_{n}\left(t_{0}\right)=\tau_{k}^{(l)} / \sqrt{\Omega_{0}}, l=1,2, \ldots, k$, we see that the equation of quantum trajectories coincide with Eq. (42) of moving zeros. That is, along such "ghost" trajectories the probability density $\rho_{n, k}$ vanishes and the corresponding quantum potential is singular. However, for properly chosen initial conditions, one can obtain quantum trajectories, which indeed satisfy the two important properties: they don't cross and don't pass through nodes (zeros of the wave function), [2,4].

\section{Laguerre type Madelung fluid and complex Burgers equation}

The Sturm-Liouville problem for the associated Laguerre polynomials is defined by the differential equation $d\left(t^{m+1} e^{-t} \dot{x}\right) / d t+\lambda t^{m} e^{-t} x=0,0<t<\infty, m>-1$, and boundary conditions that the eigenfunctions must be finite at $t=0$, and they must be of polynomial order as $t \rightarrow \infty$. Then, the eigenvalues are $\lambda=n, n=0,1,2, \ldots$ and the corresponding eigenfunctions are the associated Laguerre polynomials $L_{n}^{m}(t)=$ $e^{t} t^{-m}(n !)^{-1} d^{n}\left(e^{-t} t^{n+m}\right) / d x^{n}$. It follows that $L_{n}^{m}(t)$ are solutions of the classical damped parametric oscillator

$$
\ddot{x}+\frac{(m+1-t)}{t} \dot{x}+\frac{n}{t} x=0, \quad x\left(t_{0}\right) \neq 0, \quad \dot{x}\left(t_{0}\right)=0,
$$

with $\mu(t)=t^{m+1} e^{-t}$, damping coefficient $\Gamma(t)=(m+1-t) / t$, frequency $\omega^{2}(t)=$ $n / t$. Then the corresponding Laguerre type Schrödinger equation

$$
i \hbar \frac{\partial \Psi}{\partial t}=-\frac{\hbar^{2} e^{t}}{2 t^{m+1}} \frac{\partial^{2}}{\partial q^{2}} \Psi+\frac{n t^{m}}{2 e^{t}} q^{2} \Psi, \quad m>-1, \quad n=0,1,2, \ldots,
$$

with specific initial conditions $\Psi\left(q, t_{0}\right)=\varphi_{k}(q), k=0,1,2, \ldots$, has exact solutions, [27],

$$
\begin{aligned}
\Psi_{n, k}^{m}(q, t)= & N_{k} \sqrt{R_{n}^{m}(t)} \times \exp \left(\frac{i}{2 \hbar}\left(t^{m+1} e^{-t} \frac{\dot{L}_{n}^{m}(t)}{L_{n}^{m}(t)}\right) q^{2}\right) \\
& \times \exp \left(-\frac{i}{2} \Omega_{0}^{2} g_{n}^{m}(t)\left(R_{n}^{m}\right)^{2}(t) q^{2}\right)
\end{aligned}
$$




$$
\begin{aligned}
& \times \exp \left(i\left(k+\frac{1}{2}\right) \arctan \left(\Omega_{0} g_{n}^{m}(t)\right)\right) \times \exp \left(-\frac{\Omega_{0}}{2}\left(R_{n}^{m}\right)^{2}(t) q^{2}\right) \\
& \times H_{k}\left(\sqrt{\Omega_{0}} R_{n}^{m}(t) q\right), \quad k=0,1,2, \ldots,
\end{aligned}
$$

where

$$
\begin{aligned}
& g_{n}^{m}(t)=-\hbar\left(L_{n}^{m}\left(t_{0}\right)\right)^{2} \int^{t} \frac{e^{\xi} d \xi}{\xi^{m+1}\left(L_{n}^{m}\right)^{2}(\xi)}, \quad g\left(t_{0}\right)=0 ; \\
& R_{n}^{m}(t)=\left(\frac{\left(L_{n}^{m}\right)^{2}\left(t_{0}\right)}{\left(L_{n}^{m}\right)^{2}(t)+\left[\Omega_{0} g_{n}^{m}(t) L_{n}^{m}(t)\right]^{2}}\right)^{\frac{1}{2}} .
\end{aligned}
$$

Using Madelung representation we get the system of generalized Hamilton-Jocobi equations

$$
\left\{\begin{array}{l}
\frac{\partial S}{\partial t}+\frac{e^{t}}{2 t^{m+1}}\left(\frac{\partial S}{\partial q}\right)^{2}-\frac{\hbar^{2} e^{t}}{2 t^{m+1}}\left[\frac{1}{\sqrt{\rho}} \frac{\partial^{2} \sqrt{\rho}}{\partial q^{2}}\right]+\frac{n t^{m} e^{-t}}{2} q^{2}=0, \\
\frac{\partial \rho}{\partial t}+\frac{\partial}{\partial q}\left[\frac{\rho e^{t}}{t^{m+1}} \frac{\partial S}{\partial q}\right]=0,
\end{array}\right.
$$

which with specific initial conditions

$S_{k}\left(q, t_{0}\right)=0, \quad \rho_{k}\left(q, t_{0}\right)=N_{k}^{2} \exp \left(-\left(\sqrt{\Omega_{0}} q\right)^{2}\right) H_{k}^{2}\left(\sqrt{\Omega_{0}} q\right), \quad k=0,1,2, \ldots$ has exact solutions

$$
\begin{aligned}
S_{n, k}^{m}(q, t)= & \frac{1}{2}\left(t^{m+1} e^{-t} \frac{\dot{L}_{n}^{m}(t)}{L_{n}^{m}(t)}-\hbar \Omega_{0}^{2} g_{n}^{m}(t)\left(R_{n}^{m}\right)^{2}(t)\right) q^{2} \\
& +\hbar\left(k+\frac{1}{2}\right) \arctan \left(\Omega_{0} g_{n}^{m}(t)\right), \\
\rho_{n, k}^{m}(q, t)= & N_{k}^{2} \times R_{n}^{m}(t) \times \exp \left(-\left(\sqrt{\Omega_{0}} R_{n}^{m}(t) q\right)^{2}\right) \times H_{k}^{2}\left(\sqrt{\Omega_{0}} R_{n}^{m}(t) q\right) .
\end{aligned}
$$

Introducing the velocity $v(q, t)=\frac{e^{t}}{t^{m+1}} \frac{\partial S}{\partial q}$ in (47) gives the Madelung fluid equations

$$
\left\{\begin{array}{l}
\frac{\partial v}{\partial t}+\frac{(m+1-t)}{t} v+v \frac{\partial v}{\partial q}=-\frac{e^{t}}{t^{m+1}} \frac{\partial}{\partial q}\left[\frac{-\hbar^{2} e^{t}}{2 t^{m+1}}\left(\frac{1}{\sqrt{\rho}} \frac{\partial^{2} \sqrt{\rho}}{\partial q^{2}}\right)+\frac{n t^{m}}{2 e^{t}} q^{2}\right] \\
\frac{\partial \rho}{\partial t}+\frac{\partial}{\partial q}[\rho v]=0 .
\end{array}\right.
$$


This system (49) with initial conditions $v_{k}\left(q, t_{0}\right)=0, \rho_{k}\left(q, t_{0}\right)=\varphi_{k}^{2}(q), k=$ $0,1,2, \ldots$, has exact solutions

$$
v_{n}^{m}(q, t)=\left(\frac{\dot{L}_{n}^{m}(t)}{L_{n}^{m}(t)}-\hbar \Omega_{0}^{2} \frac{g_{n}^{m}(t)\left(R_{n}^{m}\right)^{2}(t) e^{t}}{t^{m+1}}\right) q
$$

and $\rho_{n, k}^{m}(q, t)$ given by (48).

Next we write the IVP for the complex Burgers equation of Laguerre type

$$
\left\{\begin{array}{l}
\frac{\partial V}{\partial t}+\frac{(m+1-t)}{t} V+V \frac{\partial V}{\partial q}=i \frac{\hbar e^{t}}{2 t^{m+1}} \frac{\partial^{2} V}{\partial q^{2}}-\frac{n}{t} q \\
V_{k}\left(q, t_{0}\right)=-i \frac{\hbar e^{t_{0}}}{t_{0}{ }^{m+1}} \frac{d}{d q}\left(\ln \varphi_{k}(q)\right)=\frac{i \hbar e^{t_{0}}}{t_{0}^{m+1}}\left[\Omega_{0} q-\frac{\partial_{q} H_{k}\left(\sqrt{\Omega_{0}} q\right)}{H_{k}\left(\sqrt{\Omega_{0}} q\right)}\right] \\
k=0,1,2, \ldots
\end{array}\right.
$$

which has explicit solutions of the form

$$
\begin{aligned}
V_{n, k}^{m}(q, t)= & {\left[\frac{\dot{L}_{n}^{m}(t)}{L_{n}^{m}(t)}-\hbar \Omega_{0}^{2} \frac{g_{n}^{m}(t) e^{t}}{t^{m+1}}\left(R_{n}^{m}\right)^{2}(t)\right] q } \\
& +i \frac{\hbar e^{t}}{t^{m+1}}\left[\Omega_{0}\left(R_{n}^{m}\right)^{2}(t) q-\frac{\partial_{q} H_{k}\left(\sqrt{\Omega_{0}}\left(R_{n}^{m}\right)(t) q\right)}{H_{k}\left(\sqrt{\Omega_{0}} R_{n}^{m}(t) q\right)}\right] .
\end{aligned}
$$

From Eq. (46), we see that the solution $\Psi_{n, k}^{m}(q, t)$ of the Schrödinger equation has zeros at points where $H_{k}\left(\sqrt{\Omega_{0}} R_{n}^{m}(t) q\right)=0$, and these zeros are poles of the solution $V_{n, k}^{m}(q, t)$ for the complex Burgers equation of Laguerre type. If $\tau_{k}^{(l)}$ as before denote the zeros of the Hermite polynomial $H_{k}(\xi)$, then the motion of the zeros and poles for fixed $n=0,1,2, \ldots, m>-1$ and $k=1,2, \ldots$ is described by

$$
\begin{aligned}
q_{k}^{(l)}(t)= & \frac{\tau_{k}^{(l)}}{\sqrt{\Omega_{0}} R_{n}^{m}(t)}=\frac{\tau_{k}^{(l)}}{\sqrt{\Omega_{0}}}\left(\frac{\left(L_{n}^{m}\right)^{2}(t)+\left[\Omega_{0} g_{n}^{m}(t) L_{n}^{m}(t)\right]^{2}}{\left(L_{n}^{m}\right)^{2}\left(t_{0}\right)}\right)^{\frac{1}{2}}, \\
& l=1,2, \ldots, k
\end{aligned}
$$

Thus, using the above general results one can study Madelung models and complex Burgers equations related with the associated Laguerre polynomials for any $m>-1$. In what follows, as an example we give explicit solutions for the special case when the parameters are $\mu(t)=t e^{-t}$ and $\omega^{2}(t)=n / t, t \in(0, \infty)$, which corresponds to Laguerre polynomials with $m=0$.

A. First, we introduce the Laguerre type quantum Hamilton-Jacobi system

$$
\left\{\begin{array}{l}
\frac{\partial S}{\partial t}+\frac{e^{t}}{2 t}\left(\frac{\partial S}{\partial q}\right)^{2}+\frac{n e^{-t}}{2} q^{2}=\frac{e^{t} \hbar^{2}}{2 t}\left[\frac{1}{\sqrt{\rho}} \frac{\partial^{2} \sqrt{\rho}}{\partial q^{2}}\right], \quad n=0,1,2, \ldots \\
\frac{\partial \rho}{\partial t}+\frac{\partial}{\partial q}\left[\frac{e^{t}}{t} \rho \frac{\partial S}{\partial q}\right]=0
\end{array}\right.
$$


Using transformation (22) it converts to the Laguerre type Schrödinger equation

$$
i \hbar \frac{\partial \Psi}{\partial t}=-\frac{\hbar^{2} e^{t}}{2 t} \frac{\partial^{2}}{\partial q^{2}} \Psi+\frac{n e^{-t}}{2} q^{2} \Psi
$$

where according to (2) we have $\mu(t)=t e^{-t}$, and $\omega^{2}(t)=n / t, t \in(0, \infty)$, $n=0,1,2, \ldots$ Schrödinger equation (53) with initial conditions $\Psi\left(q, t_{0}\right)=$ $\varphi_{k}(q), k=0,1,2, \ldots$, has exact solutions

$$
\begin{aligned}
\Psi_{n, k}(q, t)= & N_{k} \sqrt{R_{n}(t)} \times \exp \left(\frac{i}{2 \hbar}\left(t e^{-t} \frac{\dot{L}_{n}(t)}{L_{n}(t)}\right) q^{2}\right) \times \exp \left(-\frac{i}{2} \Omega_{0}^{2} g_{n}(t) R_{n}^{2}(t) q^{2}\right) \\
& \times \exp \left(i\left(k+\frac{1}{2}\right) \arctan \left(\Omega_{0} g_{n}(t)\right)\right) \times \exp \left(-\frac{\Omega_{0}}{2} R_{n}^{2}(t) q^{2}\right) \\
& \times H_{k}\left(\sqrt{\Omega_{0}} R_{n}(t) q\right), \quad k=0,1,2, \ldots,
\end{aligned}
$$

where $L_{n}(t), n=0,1,2, \ldots$ are the Laguerre polynomials satisfying

$$
\ddot{L}_{n}+\frac{(1-t)}{t} \dot{L}_{n}+\frac{n}{t} L_{n}=0, \quad L_{n}\left(t_{0}\right) \neq 0, \quad \dot{L}_{n}\left(t_{0}\right)=0
$$

for proper choice of $t_{0}>0$, and the auxiliary functions are

$$
\begin{aligned}
& g_{n}(t)=-\hbar L_{n}^{2}\left(t_{0}\right) \int^{t} \frac{e^{\xi} d \xi}{\xi L_{n}^{2}(\xi)}, \quad g\left(t_{0}\right)=0 \\
& R_{n}(t)=\left(\frac{L_{n}^{2}\left(t_{0}\right)}{L_{n}^{2}(t)+\left[\Omega_{0} g_{n}(t) L_{n}(t)\right]^{2}}\right)^{\frac{1}{2}} .
\end{aligned}
$$

Therefore, system (52) subject to the specific initial conditions

$$
S_{k}\left(q, t_{0}\right)=0, \quad \rho_{k}\left(q, t_{0}\right)=N_{k}^{2} \exp \left(-\left(\sqrt{\Omega_{0}} q\right)^{2}\right) H_{k}^{2}\left(\sqrt{\Omega_{0}} q\right), \quad k=0,1,2, \ldots
$$

has exact solutions

$$
S_{n, k}(q, t)=\frac{1}{2}\left(t e^{-t} \frac{\dot{L}_{n}(t)}{L_{n}(t)}-\hbar \Omega_{0}^{2} g_{n}(t) R_{n}^{2}(t)\right) q^{2}+\hbar\left(k+\frac{1}{2}\right) \arctan \left(\Omega_{0} g_{n}(t)\right),
$$

and

$$
\rho_{n, k}(q, t)=N_{k}^{2} \times R_{n}(t) \times \exp \left(-\left(\sqrt{\Omega_{0}} R_{n}(t) q\right)^{2}\right) \times H_{k}^{2}\left(\sqrt{\Omega_{0}} R_{n}(t) q\right),
$$

where $g_{n}(t)$ and $R_{n}(t)$ are given by (56). In Fig. 3a, we illustrate the behavior of $\rho_{n, k}(q, t)$ for $n=2, k=4,\left(\hbar=\Omega_{0}=1\right)$. Since $n=2$, we take $t_{0}=2$, so that 


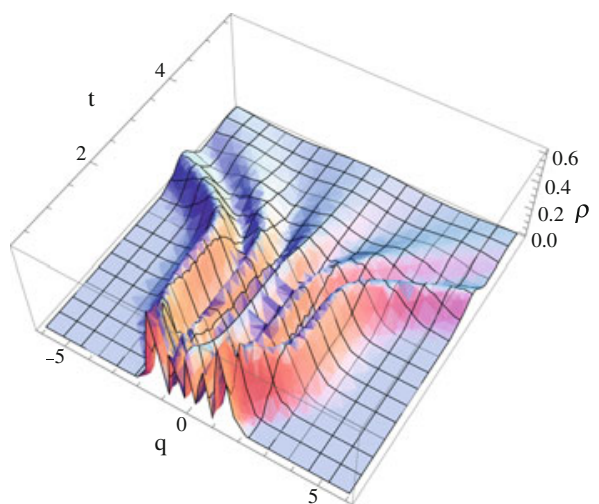

(a)

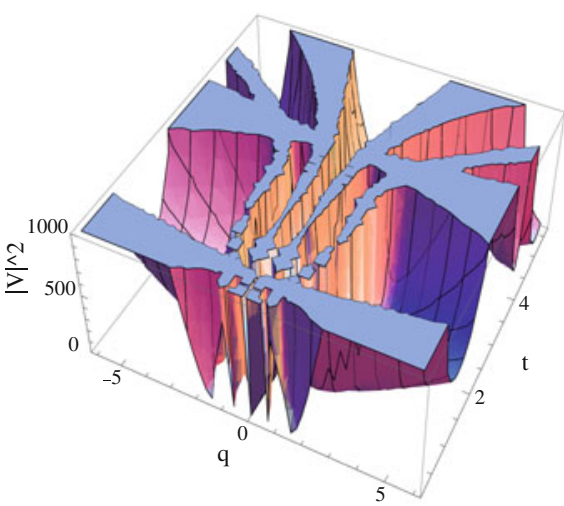

(b)

Fig. 3 Laguerre type model. a Probability density $\rho_{2,4}(q, t)$. b Plot of $\left|V_{2,4}(q, t)\right|^{2}$

the initial conditions in (55) are satisfied. Similarly, for each $n=0,1,2, \ldots$ one can always find $t_{0}>0$ such that the initial conditions hold.

B. Next, we construct a system of Laguerre type Madelung fluid equations

$$
\left\{\begin{array}{l}
\frac{\partial v}{\partial t}+\left(\frac{1-t}{t}\right) v+v \frac{\partial v}{\partial q}=-\frac{e^{t}}{t} \frac{\partial}{\partial q}\left[\frac{-\hbar^{2} e^{t}}{2 t}\left(\frac{1}{\sqrt{\rho}} \frac{\partial^{2} \sqrt{\rho}}{\partial q^{2}}\right)+\frac{n e^{-t}}{2} q^{2}\right] \\
\frac{\partial \rho}{\partial t}+\frac{\partial}{\partial q}[\rho v]=0
\end{array}\right.
$$

with variable friction coefficient $\Gamma(t)=(1-t) / t, t \in(0, \infty)$. Using transformation

$$
v(q, t)=-\frac{i \hbar e^{t}}{t} \frac{\partial}{\partial q} \ln \left(\frac{\Psi(q, t)}{|\Psi(q, t)|}\right), \quad \rho(q, t)=|\Psi(q, t)|^{2},
$$

it can be linearized in the form of Laguerre type Schrödinger equation (53). Then, system (58) with general initial conditions $v\left(q, t_{0}\right)=\tilde{v}(q), \rho\left(q, t_{0}\right)=\tilde{\rho}(q)$ has formal solution given by (59), where $\Psi(q, t)$ is solution of (53) with initial data $\Psi\left(q, t_{0}\right)=\sqrt{\tilde{\rho}(q)} \exp \left((i / \hbar) t_{0} e^{-t_{0}} \int^{q} \tilde{v}(\xi) d \xi\right)$.

System (58) with specific initial conditions

$v_{k}\left(q, t_{0}\right)=0, \quad \rho_{k}\left(q, t_{0}\right)=N_{k}^{2} \exp \left(-\left(\sqrt{\Omega_{0}} q\right)^{2}\right) H_{k}^{2}\left(\sqrt{\Omega_{0}} q\right), \quad k=0,1,2, \ldots$,

has exact solutions

$$
v_{n, k}(q, t)=\left(\frac{\dot{L}_{n}(t)}{L_{n}(t)}-\hbar \Omega_{0}^{2} \frac{e^{t} g_{n}(t) R_{n}^{2}(t)}{t}\right) q, \quad \forall k=0,1,2, \ldots
$$

and $\rho_{n, k}(q, t)$ given by (57). 
C. The potential complex Burgers equation of Laguerre type

$$
\frac{\partial F}{\partial t}+\left(\frac{1-t}{t}\right) F+\frac{1}{2}\left(\frac{\partial F}{\partial q}\right)^{2}+\frac{n}{2 t} q^{2}=\frac{i \hbar e^{t}}{2 t} \frac{\partial^{2} F}{\partial q^{2}}, \quad n=0,1,2, \ldots
$$

using the transformation

$$
F(q, t)=-\frac{i \hbar e^{t}}{t}(\ln \Psi(q, t))
$$

converts to Schrödinger equation (53). Hence, Eq. (60) with general initial condition $F\left(q, t_{0}\right)=\widetilde{F}(q)$ has solution of the form (61), where $\Psi(q, t)$ is a solution of (53) with initial condition $\Psi\left(q, t_{0}\right)=\exp \left((i / \hbar) t_{0} e^{-t_{0}} \widetilde{F}(q)\right)$. Then, it is not difficult to see that Eq. (60) with initial condition

$$
F_{k}\left(q, t_{0}\right)=\frac{i \hbar e^{t_{0}}}{t_{0}}\left(\frac{\Omega_{0}}{2} q^{2}-\ln \left(N_{k} H_{k}\left(\sqrt{\Omega_{0}} q\right)\right)\right), \quad k=0,1,2, \ldots
$$

has exact solution

$$
\begin{aligned}
F_{n, k}(q, t)= & {\left[\frac{1}{2}\left(\frac{\dot{L}_{n}(t)}{L_{n}(t)}-\hbar \Omega_{0}^{2} \frac{g_{n}(t) e^{t}}{t} R_{n}^{2}(t)\right) q^{2}+\frac{\hbar e^{t}}{t}\left(k+\frac{1}{2}\right) \arctan \left(\Omega_{0} g(t)\right)\right] } \\
& +i \frac{\hbar e^{t}}{t}\left[\frac{\Omega_{0}}{2} R_{n}^{2}(t) q^{2}-\ln \left(N_{k} \sqrt{R_{n}(t)} H_{k}\left(\sqrt{\Omega_{0}} R_{n}(t) q\right)\right)\right] .
\end{aligned}
$$

D. The Laguerre type complex Burgers equation is of the form

$$
\frac{\partial V}{\partial t}+\left(\frac{1-t}{t}\right) V+V \frac{\partial V}{\partial q}+\frac{n}{t} q=i \frac{\hbar e^{t}}{2 t} \frac{\partial^{2} V}{\partial q^{2}}, \quad n=0,1,2, \ldots
$$

Using the complex Cole-Hopf transformation

$$
V(q, t)=-\frac{i \hbar e^{t}}{t} \frac{\partial}{\partial q}(\ln \Psi(q, t)), \quad t>0
$$

it is linearizable in the form of Schrödinger equation (53). Then, Eq. (63) with general initial condition $V\left(q, t_{0}\right)=\widetilde{V}(q)$ has solution given by (64), where $\Psi(q, t)$ is solution of (53) with initial function $\Psi\left(q, t_{0}\right)=\exp \left((i / \hbar) t_{0} e^{-t_{0}} \int^{q} \widetilde{V}(\xi) d \xi\right)$. The IVP with special initial condition

$$
\left\{\begin{array}{l}
\frac{\partial V}{\partial t}+\left(\frac{1-t}{t}\right) V+V \frac{\partial V}{\partial q}+\frac{n}{t} q=i \frac{\hbar e^{t}}{2 t} \frac{\partial^{2} V}{\partial q^{2}}, \quad n=0,1,2, \ldots \\
V_{k}\left(q, t_{0}\right)=-i \frac{\hbar e^{t_{0}}}{t_{0}} \frac{d}{d q}\left(\ln \varphi_{k}(q)\right)=\frac{i \hbar e^{t_{0}}}{t_{0}}\left[\Omega_{0} q-\frac{\partial_{q} H_{k}\left(\sqrt{\Omega_{0}} q\right)}{H_{k}\left(\sqrt{\Omega_{0}} q\right)}\right] \\
k=0,1,2, \ldots
\end{array}\right.
$$


has exact solution

$$
\begin{aligned}
V_{n, k}(q, t)= & {\left[\frac{\dot{L}_{n}(t)}{L_{n}(t)}-\hbar \Omega_{0}^{2} \frac{g_{n}(t) e^{t}}{t} R_{n}^{2}(t)\right] q } \\
& +i \frac{\hbar e^{t}}{t}\left[\Omega_{0} R_{n}^{2}(t) q-\frac{\partial_{q} H_{k}\left(\sqrt{\Omega_{0}} R_{n}(t) q\right)}{H_{k}\left(\sqrt{\Omega_{0}} R_{n}(t) q\right)}\right] .
\end{aligned}
$$

We note that, for $t>0$, the function $V_{n, k}(q, t)$ has shock like singularities at finite times where $L_{n}(t)=0$, and it has moving singularities at $H_{k}\left(\sqrt{\Omega_{0}} R_{n}(t) q\right)=0$.

The complex velocity function written in the form $V(q, t)=v(q, t)+i u(q, t)$ splits the complex Burgers equation into the coupled system

$$
\left\{\begin{array}{l}
\frac{\partial v}{\partial t}+\left(\frac{1-t}{t}\right) v+v \frac{\partial v}{\partial q}-u \frac{\partial u}{\partial q}+\frac{n}{t} q=-\frac{\hbar e^{t}}{2 t} \frac{\partial^{2} u}{\partial q^{2}} \\
\frac{\partial u}{\partial t}+\left(\frac{1-t}{t}\right) u+u \frac{\partial v}{\partial q}+v \frac{\partial u}{\partial q}=\frac{\hbar e^{t}}{2 t} \frac{\partial^{2} v}{\partial q^{2}}
\end{array}\right.
$$

which with initial conditions

$$
v_{k}\left(q, t_{0}\right)=0, \quad u_{k}\left(q, t_{0}\right)=\frac{\hbar e^{t_{0}}}{t_{0}}\left[\Omega_{0} q-\frac{\partial_{q} H_{k}\left(\sqrt{\Omega_{0}} q\right)}{H_{k}\left(\sqrt{\Omega_{0}} q\right)}\right], \quad k=0,1,2, \ldots
$$

has exact solutions $v_{n}(q, t)$ and $u_{n, k}(q, t)$, the real and imaginary parts of (66), respectively. We note the relation between the different variables

$$
V(q, t)=v(q, t)+i u(q, t)=\frac{e^{t}}{t} \frac{\partial S}{\partial q}-i \frac{\hbar e^{t}}{2 t} \frac{\partial}{\partial q}(\ln \rho) .
$$

E. Solution $\Psi_{n, k}(q, t)$ of Schrödinger Eq. (54) has zeros at points where $H_{k}\left(\sqrt{\Omega_{0}} R_{n}(t) q\right)=0$, and these zeros are pole singularities of the solution $V_{n, k}(q, t)$ given by (66). Then the motion of the zeros and poles for fixed $n=0,1,2, \ldots$ and $k=1,2, \ldots$ is described by

$$
\begin{aligned}
q_{k}^{(l)}(t) & =\frac{\tau_{k}^{(l)}}{\sqrt{\Omega_{0}} R_{n}(t)}=\frac{\tau_{k}^{(l)}}{\sqrt{\Omega_{0}}}\left(\frac{L_{n}^{2}(t)+\left[\Omega_{0} g_{n}(t) L_{n}(t)\right]^{2}}{L_{n}^{2}\left(t_{0}\right)}\right)^{\frac{1}{2}}, \quad t>0, \\
l & =1,2, \ldots, k
\end{aligned}
$$

Similarly to the Hermite case, near time $t=0$, singularities show oscillatory motion and when $t \rightarrow \infty$ one has $\left|q_{k}^{(l)}(t)\right| \rightarrow \infty$. As an example, in Fig. 3 one can see the behavior of $\rho_{n, k}(q, t)$ and $\left|V_{n, k}(q, t)\right|^{2}$ for $n=2, k=4,\left(\hbar=\Omega_{0}=1\right)$. In Fig. 3b we observe that, at zeros of $L_{2}(t)$ there are finite time singularities, and also since $k=4$, there are four moving zeros of $\rho_{2,4}(q, t)$, which are also the moving poles of $V_{2,4}(q, t)$. 


\section{Jacobi type Madelung models}

The Sturm-Liouville problem for the Jacobi polynomials is defined by the differential equation $d\left[(1-t)^{\alpha+1}(1+t)^{\beta+1} \dot{x}\right] / d t+\lambda(1-t)^{\alpha}(1+t)^{\beta} x=0,-1<$ $t<1, \alpha, \beta>-1$, and boundary conditions that the eigenfunctions are finite at the singular points $t= \pm 1$. Then the eigenvalues are $\lambda=n(n+\alpha+\beta+1)$, $n=0,1,2, \ldots$, and the corresponding eigenfunctions are the Jacobi polynomials

$$
P_{n}^{\alpha, \beta}(t)=\frac{(-1)^{n}}{2^{n} n !}(1-t)^{-\alpha}(1+t)^{-\beta} \frac{d^{n}}{d t^{n}}\left[(1-t)^{\alpha+n}(1+t)^{\beta+n}\right] .
$$

Clearly, Jacobi polynomials $P_{n}^{\alpha, \beta}$ satisfy the oscillator equation

$\ddot{x}+\frac{(\beta-\alpha-(\alpha+\beta+2) t)}{1-t^{2}} \dot{x}+\frac{n(n+\alpha+\beta+1)}{1-t^{2}} x=0, \quad x\left(t_{0}\right) \neq 0, \quad \dot{x}\left(t_{0}\right)=0$,

for proper choice of $t_{0}$, where $\mu(t)=(1-t)^{\alpha+1}(1+t)^{\beta+1}, \Gamma(t)=[(\beta-\alpha-(\alpha+\beta$ $+2) t)] /\left(1-t^{2}\right)$, and frequency is $\omega^{2}(t)=[n(n+\alpha+\beta+1)] /\left(1-t^{2}\right),-1<t<1$. Therefore, solutions of the Jacobi type Schrödinger equation

$$
i \hbar \frac{\partial \Psi}{\partial t}=-\frac{\hbar^{2}}{2(1-t)^{\alpha+1}(1+t)^{\beta+1}} \frac{\partial^{2} \Psi}{\partial q^{2}}+\frac{[n(n+\alpha+\beta+1)](1-t)^{\alpha}(1+t)^{\beta}}{2} q^{2} \Psi
$$

can be obtained in terms of the Jacobi polynomials, and when the initial condition is given as $\Psi\left(q, t_{0}\right)=\varphi_{k}(q), \quad k=0,1,2, \ldots$ it has exact solutions

$$
\begin{aligned}
\Psi_{n, k}^{\alpha, \beta}(q, t)= & N_{k} \sqrt{R_{n}^{\alpha, \beta}(t)} \times \exp \left(\frac{i}{2 \hbar}\left((1-t)^{\alpha+1}(1+t)^{\beta+1} \frac{\dot{P}_{n}^{\alpha, \beta}(t)}{P_{n}^{\alpha, \beta}(t)}\right) q^{2}\right) \\
& \times \exp \left(-\frac{i}{2} \Omega_{0}^{2} g_{n}^{\alpha, \beta}(t)\left(R_{n}^{\alpha, \beta}\right)^{2}(t) q^{2}\right) \exp \left(i\left(k+\frac{1}{2}\right) \arctan \left(\Omega_{0} g_{n}^{\alpha, \beta}(t)\right)\right) \\
& \times \exp \left(-\frac{\Omega_{0}}{2}\left(R_{n}^{\alpha, \beta}(t)\right)^{2} q^{2}\right) H_{k}\left(\sqrt{\Omega_{0}} R_{n}^{\alpha, \beta}(t) q\right), \quad k=0,1,2, \ldots,
\end{aligned}
$$

where the auxiliary functions are

$$
\begin{aligned}
& g_{n}^{\alpha, \beta}(t)=-\hbar\left(P_{n}^{\alpha, \beta}\left(t_{0}\right)\right)^{2} \int^{t} \frac{d \xi}{(1-\xi)^{\alpha+1}(1+\xi)^{\beta+1}\left(P_{n}^{\alpha, \beta}(\xi)\right)^{2}}, \quad g\left(t_{0}\right)=0 \\
& R_{n}^{\alpha, \beta}(t)=\left(\frac{\left(P_{n}^{\alpha, \beta}\left(t_{0}\right)\right)^{2}}{P_{n}^{\alpha, \beta}(t)^{2}+\left[\Omega_{0} g_{n}^{\alpha, \beta}(t) H_{n}(t)\right]^{2}}\right)^{\frac{1}{2}}
\end{aligned}
$$


Madelung representation of the complex-valued wave function decomposes the Eq. (68) into the generalized Hamilton-Jacobi system

$$
\left\{\begin{array}{l}
\frac{\partial S}{\partial t}+\frac{1}{2(1-t)^{\alpha+1}(1+t)^{\beta+1}}\left(\frac{\partial S}{\partial q}\right)^{2}-\frac{\hbar^{2}}{2(1-t)^{\alpha+1}(1+t)^{\beta+1}}\left[\frac{1}{\sqrt{\rho}} \frac{\partial^{2} \sqrt{\rho}}{\partial q^{2}}\right] \\
+\frac{n(n+\alpha+\beta+)(1-t)^{\alpha}(1+t)^{\beta}}{2} q^{2}=0, \\
\frac{\partial \rho}{\partial t}+\frac{\partial}{\partial q}\left[\frac{\rho}{(1-t)^{\alpha+1}(1+t)^{\beta+1}} \frac{\partial S}{\partial q}\right]=0,
\end{array}\right.
$$

which with specific initial conditions

$$
S_{k}\left(q, t_{0}\right)=0, \quad \rho_{k}\left(q, t_{0}\right)=N_{k}^{2} \exp \left(-\left(\sqrt{\Omega_{0}} q\right)^{2}\right) H_{k}^{2}\left(\sqrt{\Omega_{0}} q\right), \quad k=0,1,2, \ldots
$$

has exact solutions

$$
\begin{aligned}
S_{n, k}^{\alpha, \beta}= & \frac{1}{2}\left((1-t)^{\alpha+1}(1+t)^{\beta+1} \frac{\dot{P}_{n}^{\alpha, \beta}(t)}{P_{n}^{\alpha, \beta}(t)}-\hbar \Omega_{0}^{2} g_{n}^{\alpha, \beta}(t)\left(R_{n}^{\alpha, \beta}\right)^{2}(t)\right) q^{2} \\
& +\hbar\left(k+\frac{1}{2}\right) \arctan \left(\Omega_{0} g_{n}^{\alpha, \beta}(t)\right),
\end{aligned}
$$

and

$\rho_{n, k}^{\alpha, \beta}(q, t)=N_{k}^{2} \times R_{n}^{\alpha, \beta}(t) \times \exp \left(-\left(\sqrt{\Omega_{0}} R_{n}^{\alpha, \beta}(t) q\right)^{2}\right) \times H_{k}^{2}\left(\sqrt{\Omega_{0}} R_{n}^{\alpha, \beta}(t) q\right)$.

Introducing classical velocity, $v(q, t)=\frac{1}{(1-t)^{\alpha+1}(1+t)^{\beta+1}} \frac{\partial S}{\partial q}$ in system (71), we obtain the Madelung hydrodynamic equations

$$
\left\{\begin{array}{l}
\frac{\partial v}{\partial t}+\frac{(\beta-\alpha-(\alpha+\beta+2) t)}{1-t^{2}} v+v \frac{\partial v}{\partial q}=\frac{-1}{(1-t)^{\alpha+1}(1+t)^{\beta+1}} \\
\times \frac{\partial}{\partial q}\left[\frac{-\hbar^{2}}{2(1-t)^{\alpha+1}(1+t)^{\beta+1}}\left(\frac{1}{\sqrt{\rho}} \frac{\partial^{2} \sqrt{\rho}}{\partial q^{2}}\right)+\frac{n(n+\alpha+\beta+1)(1-t)^{\alpha}(1+t)^{\beta}}{2} q^{2}\right] \\
\frac{\partial \rho}{\partial t}+\frac{\partial}{\partial q}[\rho v]=0
\end{array}\right.
$$

which with initial conditions $v_{k}\left(q, t_{0}\right)=0, \rho_{k}\left(q, t_{0}\right)=\varphi_{k}^{2}(q), \quad k=0,1,2, \ldots$, has exact solutions

$$
v_{n}^{\alpha, \beta}(q, t)=\left(\frac{\dot{P}_{n}^{\alpha, \beta}(t)}{P_{n}^{\alpha, \beta}(t)}-\hbar \Omega_{0}^{2} \frac{g_{n}^{\alpha, \beta}(t)\left(R_{n}^{\alpha, \beta}\right)^{2}(t)}{(1-t)^{\alpha+1}(1+t)^{\beta+1}}\right) q, \quad \forall k
$$

and $\rho_{n, k}^{\alpha, \beta}(q, t)$ as found before. 
The IVP for the complex Burgers equation of Jacobi type

$$
\left\{\begin{array}{l}
\frac{\partial V}{\partial t}+\frac{(\beta-\alpha-(\alpha+\beta+2) t)}{1-t^{2}} V+V \frac{\partial V}{\partial q}=\frac{i \hbar}{2(1-t)^{\alpha+1}(1+t)^{\beta+1}} \frac{\partial^{2} V}{\partial q^{2}} \\
-\frac{n(n+\alpha+\beta+1)}{1-t^{2}} q \\
V_{k}\left(q, t_{0}\right)=\frac{i \hbar}{\left(1-t_{0}\right)^{\alpha+1}\left(1+t_{0}\right)^{\beta+1}}\left[\Omega_{0} q-\frac{\partial_{q} H_{k}\left(\sqrt{\Omega_{0}} q\right)}{H_{k}\left(\sqrt{\Omega_{0}} q\right)}\right], \quad k=0,1,2, \ldots
\end{array}\right.
$$

are explicitly found as

$$
\begin{aligned}
& V_{n, k}^{\alpha, \beta}(q, t)=\left[\frac{\dot{P}_{n}^{\alpha, \beta}(t)}{P_{n}^{\alpha, \beta}(t)}-\hbar \Omega_{0}^{2} \frac{g_{n}^{\alpha, \beta}(t)}{(1-t)^{\alpha+1}(1+t)^{\beta+1}}\left(R_{n}^{\alpha, \beta}\right)^{2}(t)\right] q \\
& +\frac{i \hbar}{(1-t)^{\alpha+1}(1+t)^{\beta+1}}\left[\Omega_{0}\left(R_{n}^{\alpha, \beta}\right)^{2}(t) q-\frac{\partial_{q} H_{k}\left(\sqrt{\Omega_{0}} R_{n}^{\alpha, \beta}(t) q\right)}{H_{k}\left(\sqrt{\Omega_{0}} R_{n}^{\alpha, \beta}(t) q\right)}\right] .
\end{aligned}
$$

From Eq. (69), we see that the solution $\Psi_{n, k}^{\alpha, \beta}(q, t)$ of the Schrödinger equation has zeros at points where $H_{k}\left(\sqrt{\Omega_{0}} R_{n}^{\alpha, \beta}(t) q\right)=0$, and these are the poles of the solution $V_{n, k}^{\alpha, \beta}(q, t)$. If $\tau_{k}^{(l)}$ are the zeros of the Hermite polynomial $H_{k}(\xi), l=1,2, \ldots, k$, then the motion of the zeros and poles for fixed $n, \alpha, \beta$ and each $k=1,2, \ldots$ is described by

$$
\begin{gathered}
q_{k}^{(l)}(t)=\frac{\tau_{k}^{(l)}}{\sqrt{\Omega_{0}} R_{n}^{\alpha, \beta}(t)}=\frac{\tau_{k}^{(l)}}{\sqrt{\Omega_{0}}}\left(\frac{\left(P_{n}^{\alpha, \beta}(t)\right)^{2}+\left[\Omega_{0} g_{n}^{\alpha, \beta}(t) P_{n}^{\alpha, \beta}(t)\right]^{2}}{\left(P_{n}^{\alpha, \beta}\left(t_{0}\right)\right)^{2}}\right)^{\frac{1}{2}}, \\
l=1,2, \ldots, k .
\end{gathered}
$$

In what follows, as an example we consider Madelung fluid and complex Burgers equation related with the first-kind Chebyshev (FKC) polynomials, which correspond to the case $\alpha=\beta=-1 / 2$, and parameters of the form $\mu(t)=\sqrt{1-t^{2}}$, and $\omega^{2}(t)=n^{2} /\left(1-t^{2}\right), t \in(-1,1)$.

A. The FKC-type quantum Hamilton-Jacobi system is of the form

$$
\left\{\begin{array}{l}
\frac{\partial S}{\partial t}+\frac{1}{2 \sqrt{1-t^{2}}}\left(\frac{\partial S}{\partial q}\right)^{2}+\frac{n^{2}}{2 \sqrt{1-t^{2}}} q^{2}=\frac{\hbar^{2}}{2 \sqrt{1-t^{2}}}\left[\frac{1}{\sqrt{\rho}} \frac{\partial^{2} \sqrt{\rho}}{\partial q^{2}}\right] \\
n=0,1,2, \ldots, \\
\frac{\partial \rho}{\partial t}+\frac{\partial}{\partial q}\left[\frac{\rho}{\sqrt{1-t^{2}}} \frac{\partial S}{\partial q}\right]=0 .
\end{array}\right.
$$


With general initial conditions $S\left(q, t_{0}\right)=\tilde{S}(q), \rho\left(q, t_{0}\right)=\tilde{\rho}(q)$, it has formal solution

$$
S(q, t)=-i \hbar \ln \left(\frac{\Psi(q, t)}{|\Psi(q, t)|}\right), \quad \rho(q, t)=|\Psi(q, t)|^{2},
$$

where $\Psi(q, t)$ corresponds to solution of the FKC-type Schrödinger equation

$$
i \hbar \frac{\partial \Psi}{\partial t}=-\frac{\hbar^{2}}{2 \sqrt{1-t^{2}}} \frac{\partial^{2}}{\partial q^{2}} \Psi+\frac{n^{2}}{2 \sqrt{1-t^{2}}} q^{2} \Psi, \quad n=0,1,2, \ldots
$$

with initial condition $\Psi\left(q, t_{0}\right)=\sqrt{\tilde{\rho}(q)} \exp \left(\frac{i}{\hbar} \tilde{S}(q)\right)$. In particular, if $\Psi\left(q, t_{0}\right)=$ $\varphi_{k}(q), \quad k=0,1,2, \ldots$, Eq. (79) has exact solutions

$$
\begin{aligned}
\Psi_{n, k}(q, t)= & N_{k} \sqrt{R_{n}(t)} \times \exp \left(\frac{i}{2 \hbar}\left(\sqrt{1-t^{2}} \frac{\dot{T}_{n}(t)}{T_{n}(t)}\right) q^{2}\right) \times \exp \left(-\frac{i}{2} \Omega_{0}^{2} g_{n}(t) R_{n}^{2}(t) q^{2}\right) \\
& \times \exp \left(i\left(k+\frac{1}{2}\right) \arctan \left(\Omega_{0} g_{n}(t)\right)\right) \times \exp \left(-\frac{\Omega_{0}}{2} R_{n}^{2}(t) q^{2}\right) \\
& \times H_{k}\left(\sqrt{\Omega_{0}} R_{n}(t) q\right), \quad k=0,1,2, \ldots,
\end{aligned}
$$

where $T_{n}(t), n=0,1,2, \ldots$ are the first-kind Chebyshev polynomials satisfying

$$
\ddot{T}_{n}-\frac{t}{1-t^{2}} \dot{T}_{n}+\frac{n^{2}}{1-t^{2}} T_{n}=0, \quad T_{n}\left(t_{0}\right) \neq 0, \quad \dot{T}_{n}\left(t_{0}\right)=0, \quad t_{0} \in(-1,1),
$$

and the auxiliary functions are

$$
\begin{aligned}
& g_{n}(t)=-\hbar T_{n}^{2}\left(t_{0}\right) \int^{t} \frac{d \xi}{\sqrt{1-t^{2}} T_{n}^{2}(\xi)}, \quad g\left(t_{0}\right)=0 \\
& R_{n}(t)=\left(\frac{T_{n}^{2}\left(t_{0}\right)}{T_{n}^{2}(t)+\left[\Omega_{0} g_{n}(t) T_{n}(t)\right]^{2}}\right)^{\frac{1}{2}}
\end{aligned}
$$

Therefore, system (77) with specific initial conditions

$S_{k}\left(q, t_{0}\right)=0, \quad \rho_{k}\left(q, t_{0}\right)=N_{k}^{2} \exp \left(-\left(\sqrt{\Omega_{0}} q\right)^{2}\right) H_{k}^{2}\left(\sqrt{\Omega_{0}} q\right), \quad k=0,1,2, \ldots$

has exact solutions

$S_{n, k}(q, t)=\frac{1}{2}\left(\sqrt{1-t^{2}} \frac{\dot{T}_{n}(t)}{T_{n}(t)}-\hbar \Omega_{0}^{2} g_{n}(t) R_{n}^{2}(t)\right) q^{2}+\hbar\left(k+\frac{1}{2}\right) \arctan \left(\Omega_{0} g_{n}(t)\right)$, 
and

$$
\rho_{n, k}(q, t)=N_{k}^{2} \times R_{n}(t) \times \exp \left(-\left(\sqrt{\Omega_{0}} R_{n}(t) q\right)^{2}\right) \times H_{k}^{2}\left(\sqrt{\Omega_{0}} R_{n}(t) q\right),
$$

where $g_{n}(t)$ and $R_{n}(t)$ are given by $(81)$.

B. Next, setting $v(q, t)=\left(1 / \sqrt{1-t^{2}}\right) \partial S / \partial q$ in system (77), we obtain FKC-type Madelung fluid equations

$$
\left\{\begin{array}{l}
\frac{\partial v}{\partial t}-\left(\frac{t}{1-t^{2}}\right) v+v \frac{\partial v}{\partial q}=-\frac{1}{\sqrt{1-t^{2}}} \frac{\partial}{\partial q}\left[\frac{-\hbar^{2}}{2 \sqrt{1-t^{2}}}\left(\frac{1}{\sqrt{\rho}} \frac{\partial^{2} \sqrt{\rho}}{\partial q^{2}}\right)+\frac{n^{2}}{2 \sqrt{1-t^{2}}} q^{2}\right] \\
\frac{\partial \rho}{\partial t}+\frac{\partial}{\partial q}[\rho v]=0 .
\end{array}\right.
$$

Using transformation

$$
v(q, t)=-\frac{i \hbar}{\sqrt{1-t^{2}}} \frac{\partial}{\partial q} \ln \left(\frac{\Psi(q, t)}{|\Psi(q, t)|}\right), \quad \rho(q, t)=|\Psi(q, t)|^{2},
$$

system (83) converts to FKC-type Schrödinger equation (79). It follows that, system (83) with general initial conditions $v\left(q, t_{0}\right)=\tilde{v}(q), \rho\left(q, t_{0}\right)=\tilde{\rho}(q)$, has formal solution given by (84), where $\Psi(q, t)$ is solution of Schrödinger equation (79) with initial condition

$$
\Psi\left(q, t_{0}\right)=\sqrt{\tilde{\rho}(q)} \exp \left(\frac{i}{\hbar} \sqrt{1-t_{0}^{2}} \int^{q} \tilde{v}(\xi) d \xi\right)
$$

Therefore, the Madelung system (83) with specific initial conditions $v_{k}\left(q, t_{0}\right)=0, \quad \rho_{k}\left(q, t_{0}\right)=N_{k}^{2} \exp \left(-\left(\sqrt{\Omega_{0}} q\right)^{2}\right) H_{k}^{2}\left(\sqrt{\Omega_{0}} q\right), \quad k=0,1,2, \ldots$

has exact solutions

$$
v_{n}(q, t)=\left(\frac{\dot{T}_{n}(t)}{T_{n}(t)}-\hbar \Omega_{0}^{2} \frac{g_{n}(t) R_{n}^{2}(t)}{\sqrt{1-t^{2}}}\right) q, \quad \forall k=0,1,2, \ldots,
$$

and $\rho_{n, k}(q, t)$ given by $(82)$.

C. The potential complex Burgers equation of FKC-type

$$
\frac{\partial F}{\partial t}-\left(\frac{t}{1-t^{2}}\right) F+\frac{1}{2}\left(\frac{\partial F}{\partial q}\right)^{2}+\frac{n^{2}}{2\left(1-t^{2}\right)} q^{2}=\frac{i \hbar}{2 \sqrt{1-t^{2}}} \frac{\partial^{2} F}{\partial q^{2}}
$$

converts to the linear Schrödinger equation (79) using the relation

$$
F(q, t)=-\frac{i \hbar}{\sqrt{1-t^{2}}}(\ln \Psi(q, t))
$$


With general initial data $F\left(q, t_{0}\right)=\widetilde{F}(q)$, it has formal solution given by (86), where $\Psi(q, t)$ is a solution of (79) with initial data $\left.\Psi\left(q, t_{0}\right)=\exp \left(i / \hbar \sqrt{1-t_{0}^{2}}\right) \widetilde{F}(q)\right)$. The potential complex Burgers (85) with specific initial conditions

$$
F_{k}\left(q, t_{0}\right)=\frac{i \hbar}{\sqrt{1-t_{0}^{2}}}\left(\frac{\Omega_{0}}{2} q^{2}-\ln \left(N_{k} H_{k}\left(\sqrt{\Omega_{0}} q\right)\right)\right), \quad k=0,1,2, \ldots
$$

has exact solutions

$$
\begin{aligned}
F_{n, k}(q, t)= & {\left[\frac{1}{2}\left(\frac{\dot{T}_{n}(t)}{T_{n}(t)}-\hbar \Omega_{0}^{2} \frac{g_{n}(t)}{\sqrt{1-t_{0}^{2}}} R_{n}^{2}(t)\right) q^{2}+\frac{\hbar}{\sqrt{1-t_{0}^{2}}}\left(k+\frac{1}{2}\right) \arctan \left(\Omega_{0} g_{n}(t)\right)\right] } \\
& +\frac{i \hbar}{\sqrt{1-t_{0}^{2}}}\left[\frac{\Omega_{0}}{2} R_{n}^{2}(t) q^{2}-\ln \left(N_{k} \sqrt{R_{n}(t)} H_{k}\left(\sqrt{\Omega_{0}} R_{n}(t) q\right)\right)\right] .
\end{aligned}
$$

D. Now, we consider the FKC-type complex Burgers equation

$$
\frac{\partial V}{\partial t}-\frac{t}{1-t^{2}} V+V \frac{\partial V}{\partial q}+\frac{n^{2}}{\sqrt{1-t^{2}}} q=i \frac{\hbar}{2 \sqrt{1-t^{2}}} \frac{\partial^{2} V}{\partial q^{2}} \quad n=0,1,2, \ldots
$$

Linearization of this equation using the generalized Cole-Hopf transform

$$
V(q, t)=-\frac{i \hbar}{\sqrt{1-t^{2}}} \frac{\partial}{\partial q}(\ln \Psi(q, t)),
$$

leads to the FKC-type Schrödinger equation (79). Then, the complex Burgers equation (88) with general initial condition $V\left(q, t_{0}\right)=\widetilde{V}(q)$, has formal solution given by (89) where $\Psi(q, t)$ is solution of the IVP for the Schrödinger equation with initial data $\Psi\left(q, t_{0}\right)=\exp \left(\frac{i}{\hbar} \sqrt{1-t_{0}^{2}} \int^{q} \widetilde{V}(\xi) d \xi\right)$.

The IVP with special initial conditions

$$
\left\{\begin{array}{l}
\frac{\partial V}{\partial t}-\frac{t}{1-t^{2}} V+V \frac{\partial V}{\partial q}+\frac{n^{2}}{\sqrt{1-t^{2}}} q=i \frac{\hbar}{2 \sqrt{1-t^{2}}} \frac{\partial^{2} V}{\partial q^{2}} \quad n=0,1,2, \ldots \\
V_{k}\left(q, t_{0}\right)=\frac{i \hbar}{\sqrt{1-t_{0}^{2}}}\left[\Omega_{0} q-\frac{\partial_{q} H_{k}\left(\sqrt{\Omega_{0}} q\right)}{H_{k}\left(\sqrt{\Omega_{0}} q\right)}\right], \quad k=0,1,2, \ldots
\end{array}\right.
$$

has exact solutions

$$
\begin{aligned}
V_{n, k}(q, t)= & {\left[\frac{\dot{T}_{n}(t)}{T_{n}(t)}-\hbar \Omega_{0}^{2} \frac{g_{n}(t)}{\sqrt{1-t^{2}}} R_{n}^{2}(t)\right] q } \\
& +i \frac{\hbar}{\sqrt{1-t^{2}}}\left[\Omega_{0} R_{n}^{2}(t) q-\frac{\partial_{q} H_{k}\left(\sqrt{\Omega_{0}} R_{n}(t) q\right)}{H_{k}\left(\sqrt{\Omega_{0}} R_{n}(t) q\right)}\right] .
\end{aligned}
$$

E. The motion of the zeros of the wave function $\Psi_{n, k}(q, t)$ given by (80), and the poles of the complex velocity $V_{n, k}(q, t)$ for fixed $n=0,1,2, \ldots$ and $k=1,2, \ldots$ is 


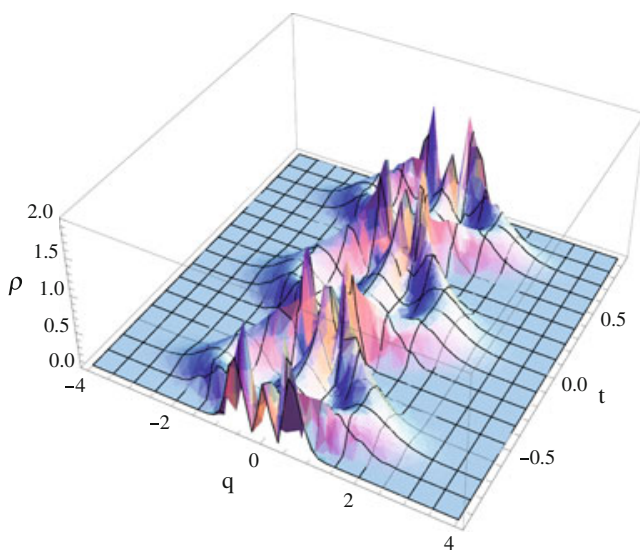

(a)

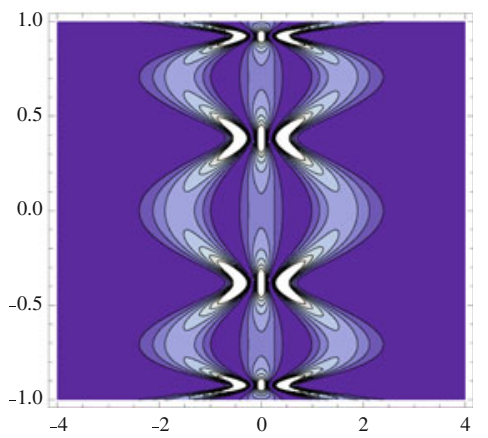

(b)

Fig. 4 FK Chebychev type model. a Plot of $\rho_{4,2}(q, t)$. b Contour plot of $\rho_{4,2}(q, t)$

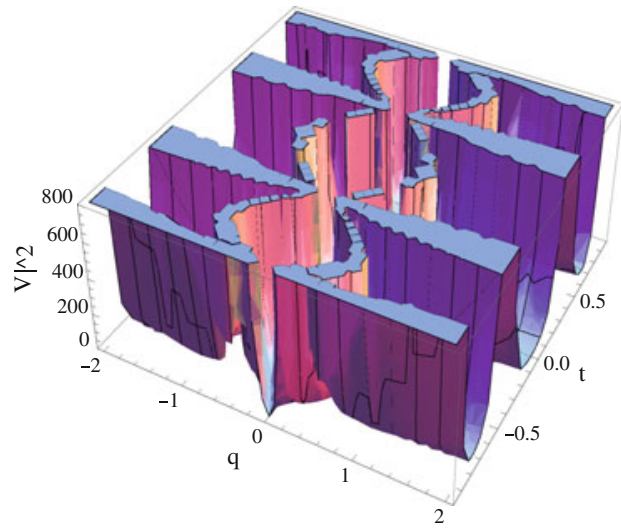

(a)

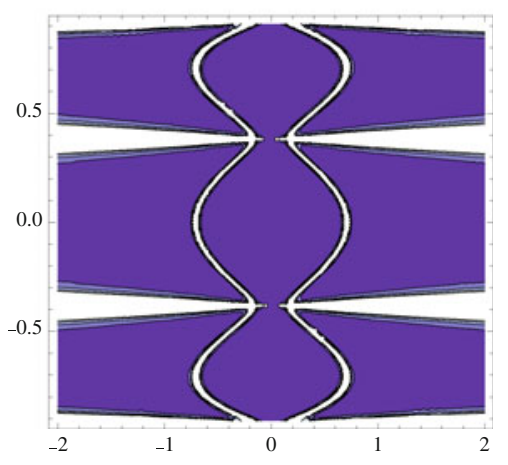

(b)

Fig. 5 FK Chebyshev type model. a Plot of $\left|V_{4,2}(q, t)\right|^{2}$. b Contour plot of $\left|V_{4,2}(q, t)\right|^{2}$

described by

$$
q_{k}^{(l)}(t)=\frac{\tau_{k}^{(l)}}{\sqrt{\Omega_{0}} R_{n}(t)}=\frac{\tau_{k}^{(l)}}{\sqrt{\Omega_{0}}}\left(\frac{T_{n}^{2}(t)+\left[\Omega_{0} g_{n}(t) T_{n}(t)\right]^{2}}{T_{n}^{2}\left(t_{0}\right)}\right)^{\frac{1}{2}}, \quad l=1,2, \ldots, k
$$

In Fig. 4, we illustrate the behavior of the probability density $\rho_{n, k}(q, t)$ and in Fig. 5 the behavior of $\left|V_{n, k}(q, t)\right|^{2}$ for the case $n=4, k=2$, $\left(\hbar=\omega_{0}=1, t_{0}=0\right)$. The oscillatory motion of the two zeros and poles, respectively is confined in time interval $(-1,1)$, and can be observed from the given corresponding contour plots. 


\section{Conclusion}

In this article we applied Quantum Sturm-Liouville problems for solving variable parametric Madelung quantum fluid models. Precisely, we have introduced and obtained exact solutions of Madelung fluid and complex Burgers equations of the Hermite, Laguerre and Jacobi (first kind Chebyshev) type. For the Hermite type of Schrödinger equation, the mass is exponentially decreasing and the square frequency is an integer constant. Corresponding Madelung fluid includes friction term linearly dependent on time and quantum potential strength with exponential growth. It determines complex Burgers equation with the similar friction, linear external potential and complex diffusion coefficient with Gaussian growth in time. The quantum trajectory picture for specifically chosen initial conditions was described explicitly. For the Laguerre type Schrödinger equation the mass term is polynomial for small times and exponentially decreasing for large times, while the frequency is decaying as inverse power of time. It gives Madelung fluid with rationally time dependent friction and exponentially growing quantum potential. Similarly to the Hermite case, near time zero, singularities of the velocity field show oscillatory motion. From the Jacobi type models, the first kind Chebyshev case was treated in details. In that case, the mass is an algebraic function of time, the friction force and the strength of quantum potential are rational, and the oscillatory motion of the zeros/poles is confined in finite interval. In a similar way, as we have explored in this paper one can study the Madelung models related with the hypergeometric function and its other degenerations.

Here, we would like to notice that for the Madelung fluid considered as an alternative formulation of the Schrödinger equation, we have solved the nonlinear initial value problem under initial conditions, meaningful for the corresponding linear Schrödinger equation. However, for the quantum hydrodynamics and complex Burgers equations, other type of initial conditions may also provide interesting and meaningful solutions. Such problems will be addressed in our later work. As we already mentioned in the Introduction part, our models were not determined from concrete physical system, but motivated by exact solvability. It provides from one side a wide class of exactly solvable models which can be used as an approximation to real systems, and from another side they can give more insights on nonlinear dynamics in dissipative quantum fluids and used as a test for improving related computational methods.

Acknowledgments This work is supported by TÜBITAK, Project No: 110T679 and Izmir Institute of Technology.

\section{References}

1. E. Madelung, Z. Phys. 40, 322 (1926)

2. P.R. Holland, The Quantum Theory of Motion (Cambridge University Press, New York, 1993)

3. P. Holland, Ann. Phys. 315, 505 (2005)

4. R.E. Wyatt, Quantum Dynamics with Trajectories (Springer, New York, 2005)

5. R. Feynman, The Feynman Lectures on Physics, Quantum Mechanics, vol. 3 (Addison-Wesley Publishing Co., London, 1965)

6. R. Feynman, Statistical Mechanics: A Set of Lectures (Westview Press, Boulder, CO, 1998) 
7. V.E. Zakharov,in Singular Limit of Dispersive Waves (NATO Adv. Sci. Inst. Ser. B Phys.), vol. 320, ed. by N.M. Ercolani et al. (New York: Plenum, 1994)

8. Sh. Jin, C.D. Levermore, D.W. McLaughlin, Comm. Pure Appl. Math. LII: 0613, 0613 (1999)

9. J.H. Lee, C.K. Lin, O.K. Pashaev, Chaos Solitons Fractals 19, 109 (2004)

10. C. Michoski, J.A. Evans, P.G. Schmitz, A. Vasseur, www.ma.utexas.edu/users/vasseur/preprints/ tQHD.pdf.

11. M. Okuyama, K. Takatsuka, Chem. Phys. Lett. 476, 109 (2009)

12. I. Barth et al., Chem. Phys. Lett. 481, 118 (2009)

13. R. Tsekov, Phys. Scr. 83, 035004 (2011)

14. P. Caldirola, Nouovo Cimento 18, 393 (1941)

15. E. Kanai, Prog. Theo. Phys. 3, 440 (1948)

16. H. Dekker, Classical and quantum mechanics of damped harmonic oscillator. Phys. Rep. 80, 1-112 (Amsterdam, North-Holland Publishing Company, 1981)

17. K. Husimi, Prog. Theor. Phys. 9, 381 (1953)

18. R. Feynman, Phys. Rev. 84, 108 (1951)

19. H.R. Lewis Jr, W.B. Riesenfeld, J. Math. Phys. 10, 1458 (1969)

20. J. Wei, E. Norman, J. Math. Phys. 4, 575 (1963)

21. A. Perelomov, Generalized Goherent States and Their Applications (Springer, Berlin, 1986)

22. I.A. Pedrosa, G.P. Serra, I. Guides I. Phys. Rev. A 56(5), 4300 (1995)

23. A. Sakharov, Zh. Eksp. Teor. Fiz. 49, 345 (1965), [Sov. Phys. JETP 22, 241 (1966)]

24. J.D. Cole, Quart. Appl. Math. 9, 225 (1951)

25. E. Hopf, Comm. Pure Appl. Math. 3, 201 (1950)

26. F. Calogero, Lett. Nuovo Cim. B 43, 177 (1978)

27. Ş.A. Büyükaşı, O.K. Pashaev, E. Ulaş-Tigrak, J. Math. Phys. 50, 072102 (2009)

28. Ş.A. Büyükaşık, O.K. Pashaev, J. Math. Phys. 51, 122108 (2010)

29. R. Courant, D. Hilbert, Methods of Mathematical Physics, vol. I (Wiley, New York, 2004) 